\title{
1 A long read optimized de novo transcriptome pipeline reveals 2 novel ocular developmentally regulated gene isoforms and disease targets
}

\section{Abstract}

\author{
Vinay S. Swamy ${ }^{1}$, Temesgen D. Fufa ${ }^{2}$, Robert B. Hufnagel2, and David M. \\ McGaughey ${ }^{1}$
}

${ }^{1}$ Bioinformatics Group, Ophthalmic Genetics \& Visual Function Branch, National Eye Institute, Institutes of Health

${ }^{2}$ Medical Genetics and Ophthalmic Genomics Unit, National Eye Institute, National Institutes of Health

Correspondence: David M. McGaughey<mcgaugheyd@mail.nih.gov>

De novo transcriptome construction from short-read RNA-seq is a common method for reconstructing mRNA transcripts within a given sample. However, the precision of this process is unclear as it is difficult to obtain a ground-truth measure of transcript expression. With advances in third generation sequencing, full length transcripts of whole transcriptomes can be accurately sequenced to generate a ground-truth transcriptome. We generated long-read PacBio and short-read Illumina RNA-seq data from a human induced pluripotent stem cell- derived retinal pigmented epithelium (iPSC-RPE) cell line. We use long-read data to identify simple metrics for assessing de novo transcriptome construction and optimize a short-read based de novo transcriptome construction pipeline. We apply this this pipeline to construct transcriptomes for 340 short-read RNA-seq samples originating from healthy adult and fetal human retina, cornea, and RPE. We identify hundreds of novel gene isoforms and examine their significance in the context of ocular development and disease. 


\section{Introduction}

The transcriptome is defined as the set of unique RNA transcripts expressed in a biological system. A single gene can have multiple distinct transcripts, or isoforms, and there are multiple biological processes that drive the formation of these isoforms including alternative promoter usage, alternative splicing, and alternative polyadenylation. Gene isoforms can have distinct and critical functions in biological processes like development, cell differentiation, and cell migration (Dykes et al., 2018), (Trapnell et al., 2010), (Mitra et al., 2020). Alternative usage of isoforms has also been implicated in multiple diseases including cancer, cardiovascular disease, Alzheimer's disease and diabetic retinopathy (Vitting-Seerup and Sandelin, 2017), (Neagoe Ciprian et al., 2002), (Mills et al., 2013), (Perrin et al., 2005).

Accurate annotation of gene isoforms is fundamental for understanding their biological impact. For example, while the Gencode human comprehensive transcript annotation (release 28) contains 82335 protein coding and 121500 noncoding transcripts across 19901 genes and 38480 pseudogenes, but this annotation is incomplete (Frankish et al., 2019), (Zhang et al., 2020). Some of the first high throughput methods to find novel gene isoforms used short-read ( 100bp) RNA-seq to identify novel exon-exon junctions and novel exon boundaries based soley on RNA-seq coverage (Nagalakshmi et al., 2008). More recently, several groups have developed specialized tools to use RNA-seq to reconstruct the whole transcriptome of a biological sample, dubbed de novo transcriptome construction (Haas et al., 2013),(Trapnell et al., 2010), (Pertea et al., 2015).

De novo transcriptome construction uses short-read RNA-seq to reconstruct fulllength mRNA transcripts. However, a large number of samples are necessary to overcome the noise and short-read lengths of this type of data. Because of increasingly inexpensive sequencing cost, datasets of the necessary size are now available. For example, one of the most comprehensive de novo transcriptome projects to date is CHESS, which uses the GTEx data set to construct de novo transcriptomes in over 9000 RNA-seq samples from 44 
54 GTEx dataset does not include samples from any ocular tissues, the CHESS database

55 remains an incomplete annotation of the human transcriptome.

56 Despite the increasing number of tools developed, there is no gold standard to

57 evaluate the precision and sensitivity of de novo transcriptome construction on real (not

58 simulated) biological data. Long-read sequencing technologies provide a potential solution

59 to this problem as long-read sequencing can capture full length transcripts and thus, can be

60 used to identify a more comprehensive range of gene isoforms. While previous iterations of

61 long-read sequencing technologies typically had higher error rates, the new PacBio Sequel

62 II system sequences long-reads as accurately as short-read based sequencing (Wenger et

63 al., 2019).

We propose that long-read based transcriptomes can serve as a ground truth for evaluating short-read based transcriptomes. In this study, we used PacBio long-read RNA sequencing to inform the construction of short-read transcriptomes. We generated PacBio long-read RNA-seq along with matched Illumina short-read RNA-seq data from a human induced pluripotent stem cell (iPSC)-differentiated retinal pigmented epithelium (RPE) cell line. We then designed a rigorous StringTie-based pipeline that maximizes the concordance between short and long-read de novo transcriptomes.

Finally, we applied this optimized pipeline to a data set containing 340 human

72 ocular tissue samples compiled from mining previously published, publicly available short-

73 read RNA-seq data (Swamy and McGaughey, 2019). We built transcriptomes for three

74 major ocular tissues: cornea, retina, and RPE, using RNA-seq data from both adult and fetal

75 tissues to create a high-quality pan-eye transcriptome. In addition to ocular samples, we

76 used a subset of the GTEx data set to construct transcriptomes for tissues in 44 other

77 locations across the body.

We used our gold-standard informed pan-eye de novo transcriptome to reveal

79 hundreds of novel gene isoforms in the eye and analyze their potential impact on ocular

80 biology and disease. We provide transcript annotation derived from our de novo

81 transcriptomes as a resource to other researchers through an R package. 
bioRxiv preprint doi: https://doi.org/10.1101/2020.08.21.261644; this version posted September 22, 2020. The copyright holder for this preprint (which was not certified by peer review) is the author/funder. This article is a US Government work. It is not subject to copyright under 17 USC 105 and is also made available for use under a CCO license.

\section{Results}

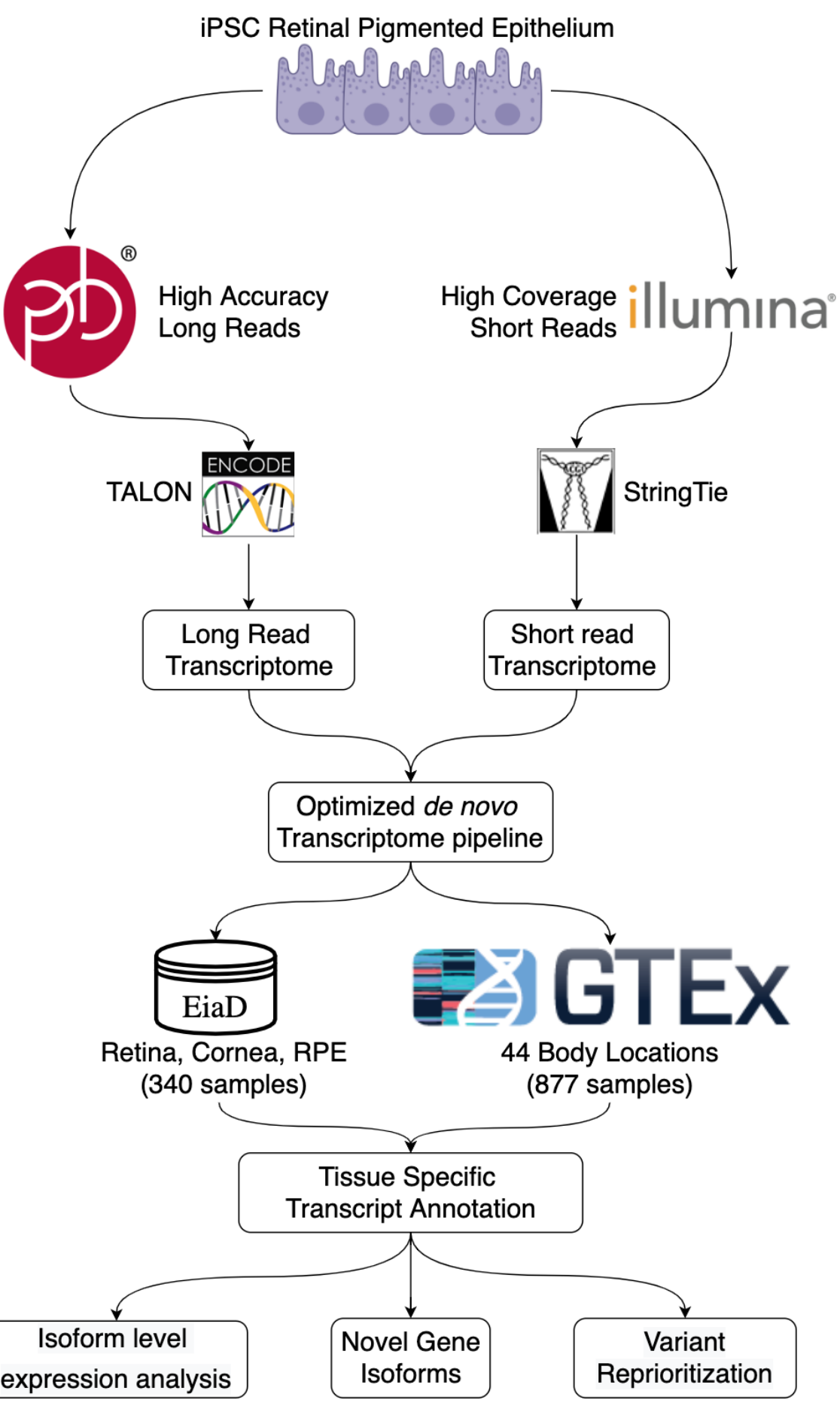
construction and analysis 


\section{Long-read PacBio RNA sequencing guides short-read de novo transcriptome} 87 construction

88 To evaluate the accuracy of short-read transcriptome construction, we first

89 generated PacBio long-read RNA-seq data and Illumina short-read RNA-seq data from iPSC-

90 RPE (Fig 1). These cells were differentiated using an optimized protocol, and thus minimal

91 biological variation is expected (Blenkinsop et al., 2015), (Maruotti et al., 2015). We used

92 these sequencing data to construct a long-read transcriptome and a short-read

93 transcriptome. In our long-read transcriptome we found 1163239 distinct transcripts, and

94 in our short-read transcriptome 366888 distinct transcripts

A

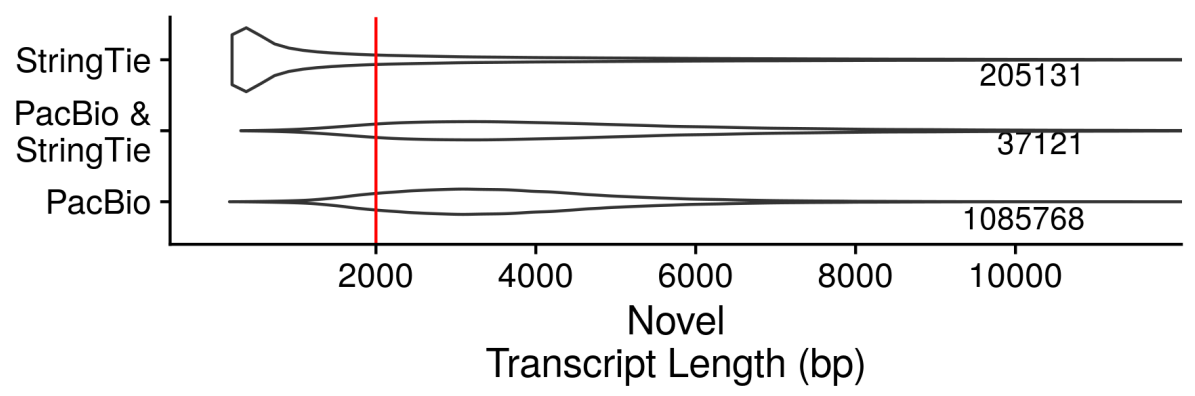

B
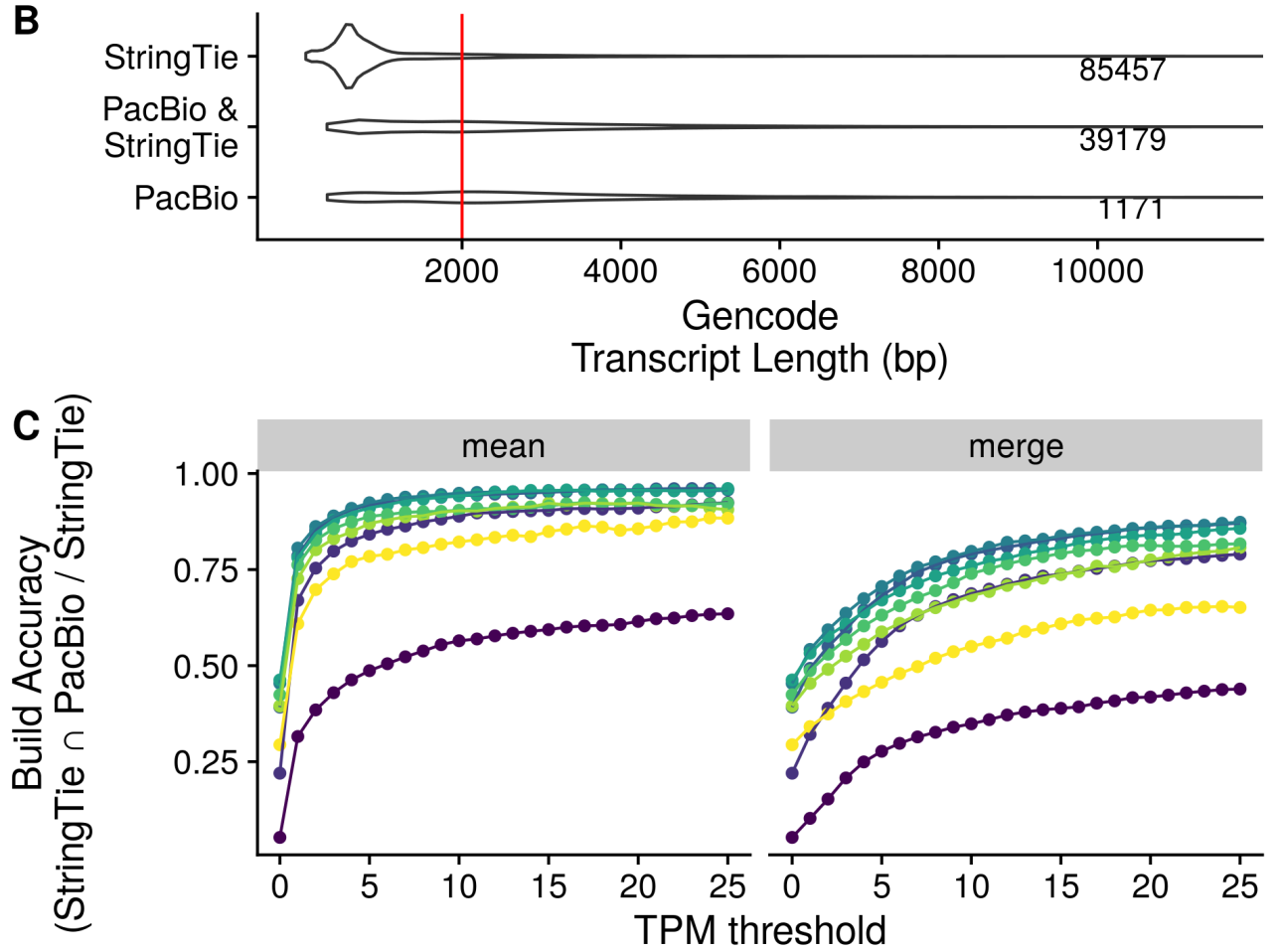

Length Interval

$\rightarrow 0-1000$

$\rightarrow 1000-2000$

$\rightarrow 2000-3000$

$\rightarrow 3000-4000$

- 4000-5000

$\rightarrow$ 5000-6000

-6000-7000

7000+ 
96

97

98

99

100

101

102

103

104

105

106

107

108

109

110

111

112

113

114

115

116

117

118

119

120

121

122

123

124

125

126

127

128

129

130

Figure 2. Transcript length and expression dictate transcriptome construction accuracy. A,B) Distributions of novel(A) and previously annotated(B) transcript lengths between PacBio (long-read) and Stringtie (short-read) transcriptomes. Each distribution is labeled with the total number of transcripts in the distribution C) short-read construction accuracy stratified by transcript length at different Transcripts Per Million (TPM)-based transcript exclusion thresholds. The "merge" method follows the protocol for constructing transcriptomes outlined by the StringTie authors and keeps any transcripts expressed above a specific TPM threshold in at least one samples. The "mean" method used by our pipeline keeps transcripts whose average expression across all samples is above a specific TPM threshold.

In our initial comparison between short and long-read transcriptomes, we noticed a low transcriptome construction accuracy (see Methods) of 0.208. When we examined the transcript lengths of each build we saw that the two methods show very different transcript length distributions for both novel and previously annotated transcripts, with the short-read build was comprised mostly of smaller transcripts (Fig 2A). As the PacBio data was generated using two different libraries for $2000 \mathrm{bp}$ and $>3000 \mathrm{bp}$ transcripts, we expected an enrichment for longer transcripts in the PacBio data set (Supplemental Figure 2). To assess accuracy relative to transcript length, we grouped transcripts by length in $1000 \mathrm{bp}$ intervals, and compared accuracy between each group. We found that accuracy significantly improves for transcripts longer than $2000 \mathrm{bp}$. The construction accuracy is 0.426 and 0.137 for transcripts above and below $2000 \mathrm{bp}$, respectively.

We experimented with various methods to remove spurious transcripts and improve construction accuracy. We first removed transcripts that were expressed $<1$ TPM in at least one sample as outlined in StringTie's recommended protocol (Pertea et al., 2016). This improved construction accuracy to 0.475 for transcripts longer than $2000 \mathrm{bp}$ and 0.212 for transcripts shorter than $2000 \mathrm{bp}$. As this accuracy was still fairly low, we tried different filtering schemes, including experimenting with machine learning-based strategies to identify transcripts that were computational artifacts (data not shown), but we found that the simplest approach with high performance was to retain transcripts that had an average TPM above a specific threshold(Fig 2C). In our downstream pipeline we keep transcripts that have at least an average of 1 TPM across all samples of the same subtissue type as this threshold achieved a build accuracy of 0.772 for transcripts longer than $2000 B p$ and retained 48470 transcripts within this short-read RPE dataset. 
131 Thousands of novel gene isoforms are detected in human subtissue-specific 132 transcriptomes

\begin{tabular}{llrrr}
\hline Tissue & Source & Samples & Studies & Transcriptome Count \\
\hline RPE & Adult & 48 & 4 & 32012 \\
RPE & Fetal & 49 & 7 & 49967 \\
Retina & Adult & 105 & 8 & 49714 \\
Retina & Fetal & 89 & 6 & 66255 \\
Cornea & Adult & 43 & 6 & 51469 \\
Cornea & Fetal & 6 & 2 & 59408 \\
\hline
\end{tabular}

133

134

135

136

137

138

139

140

141

142

143

144

145

146

147

148

149

150

151

152

153

154

155

Table 1. Ocular sample dataset overview and transcriptome count. Transcriptome count is defined as the number of unique transcripts expressed in a given tissue type

We built transcriptomes from 340 publicly available ocular tissue RNA-seq samples curated in EiaD using an efficient Snakemake pipeline (Köster and Rahmann, 2012). We included both publicly collated non-disease, non-perturbed adult and fetal samples from cornea, retina, and RPE tissues, mined from 29 different studies (Table 1). Our fetal tissues consist of both human fetal tissues and human iPSC-derived tissue, as stem cell-derived tissue has been showed to closely resemble fetal tissue. We inlcude our iPSC-RPE samples originally used to develop our pipeline within this larger set of fetal RPE samples. (Klimanskaya et al., 2004). To more accurately determine the tissue specificity of novel ocular transcripts, we supplemented our ocular data set with 877 samples from 44 body locations across 22 major tissues from the GTEx project and constructed transcriptomes for each of these body locations (GTEx Consortium et al., 2017). We refer to each distinct body location as a subtissue here after.

After initial construction of transcriptomes, we found 183442 previously annotated transcripts and 6241675 novel transcripts detected in at least one of our 1217 samples. We define a novel transcripts as all transcripts whose set of exons and introns do not exactly match that of an annotated transcript within the Gencode, Ensembl, UCSC, and Refseq annotation databases (Frankish et al., 2019), (Zerbino et al., 2018), (O’Leary et al., 2016). After using the filtering methods described above, we merged all subtissue specific transcriptomes into a single final transcriptome which contains 252983 distinct transcripts with 87592 previously annotated and 165391 novel transcripts, and includes 114.9 
156 megabases of previously unannotated genomic sequence (Table 1). We refer to the final 157 pan-body transcriptome as the DNTX annotation hereafter.

158 We split novel transcripts into two categories: novel isoforms, which are novel 159 variations of known genes, and novel loci, which are previously unreported, entirely novel 160 regions of transcribed sequence (Fig 3B). Novel isoforms are further classified by the 161 novelty of their encoded protein: isoforms with novel open reading frame, novel isoforms 162 with a known ORF, and isoforms with no ORF as noncoding isoforms (Fig 3A). The number 163 of distinct ORFs was significantly less than the number of transcripts, with 43279

164 previously annotated ORFs and 46226 novel ORFs across all subtissues. Furthermore, 165 across all subtissues there was an average of 10393 novel isoforms and 3716 novel ORFs. 


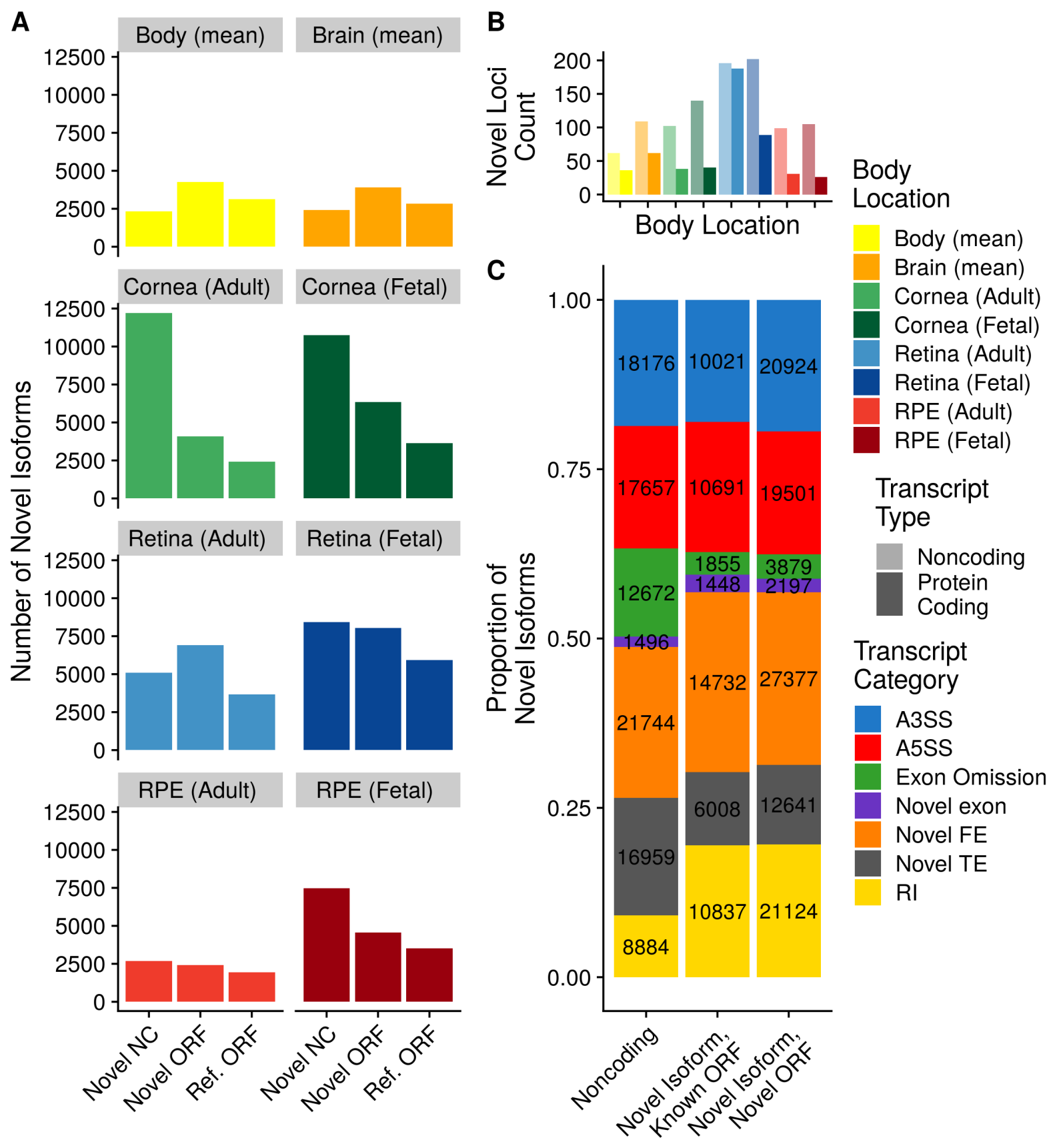

Figure 3. Overview of novel isoforms. A) Number of novel gene isoforms, grouped by transcript type. Brain and body represent an average of 13 and 34 distinct subtissues, respectively. B) Novel protein coding and noncoding loci. Novel exon composition of novel isoforms, by isoform type. Labels indicate number of transcripts. C) Classification of novel exon types, stratified by novel isoform type. 
Novel isoforms can occur due to an omission of a previously annotated exon,

174 commonly referred as exon skipping or the addition of an unannotated exon which we

175 refer to as a novel exon. We further classified novel exons by the biological process that

176 may be driving their formation: alternative promoter usage driving the addition of novel

177 first exons (FE), alternative polyadenylation driving the addition of novel terminal exons

178 (TE), and alternative splicing driving the formation of all novel exons that are not the first

179 or last exon (Landry et al., 2003), (Tian and Manley, 2017), (Wang et al., 2015). We then

180 split alternatively spliced exons into their commonly seen patterns, alternative 5' splice site

181 (A5SS), alternative 3' splice site (A3SS), and retained introns (RI). Exons whose entire

182 sequence was unannotated and is not a retained intron are fully novel exons. We note that

183 all three of these mechanisms can lead to exon skipping, so for simplicity we grouped all

184 novel isoforms resulting from exon skipping together. We found that the majority of novel

185 exons within our dataset are novel FEs. We noticed that the majority of RI exons lead to

186 novel ORFs, whereas novel isoforms with omitted exons more often lead to noncoding

187 isoforms. (Fig 3C)

De novo transcriptomes match previously published experimental data better than existing annotation

We validated de novo transcriptomes using three independent approaches. We first

191 looked for evolutionary conservation since it is commonly accepted as a proxy for

192 functional significance. We used the PhyloP 20 way species alignment, a measure of

193 conservation between species, to calculate the average conservation score for each exon in

194 the DNTX annotation and compared that to the average conservations score for each exon

195 in the Gencode annotation (Pollard et al., 2010). We found that, on average, exons in the

196 DNTX annotation are more conserved than exons in the Gencode annotation (pvalue $<2$.2e-

197 16) (Supplemental Figure 2A).

Next, since we observed an enrichment in novel first and last exons within our data

199 set, we decided to compare the TSS and TES within the DNTX annotation to two well-

200 established annotation databases from FANTOM and the polyA Atlas (Noguchi et al., 2017),

201 (Herrmann et al., 2020). We compared DNTX and Gencode TSS's to CAGE-seq data from the

202 FANTOM consortium; as CAGE-seq is optimized to detect the 5' end of transcripts, we 
203 reasoned that it can serve as a valid ground truth set to evaluate TSS detection (Takahashi

204 et al., 2012). We calculated the absolute distance of DNTX TSS's to CAGE peaks, and

205 compared them to the absolute distance of Gencode TSS's to CAGE peaks. We found that, on

206 average, DNTX TSS's were closer to CAGE peaks than Gencode TSS's (pvalue <2.2e-

207 16)(Supplemental Figure 2B).

208 Finally, we evaluated TES's using the polyA Atlas, which is comprised of

209 polyadenylation signal annotation generated from aggregating 3' seq data from multiple

210 studies. As 3'-seq data is designed to accurately capture the 3' ends of transcripts, it can

211 similarly serve as a ground truth set to evaluate the accuracy of TES's (Beck et al., 2010).

212 We calculated the absolute distance of DNTX TES's to annotated polyA signals and

213 compared them to the absolute distance of Gencode TES's to polyA signals. We found that

214 on average DNTX TES's are closer to annotated polyadenylation signals than gencode TSS's

215 (pvalue <2.2e-16) (Supplemental Figure 2C)

216 De novo transcriptomes reduce overall transcriptome sizes

217 De novo transcriptomes removed on average $76.141 \%$ of a subtissue's base

218 transcriptome. We defined base transcriptome for a subtissue as any transcript in the

219 Gencode annotation with non-zero TPM in at least one sample of a given subtissue. This

220 was a large reduction in transcriptome size and we wanted to ensure that we were not

221 unduly discarding data. We quantified transcript expression of each sample using Salmon

222 with two methods: once using the full gencode v28 human transcript annotation, and once

223 using its associated subtissue specific transcriptome. We found that despite the $76.141 \%$

224 reduction in number of transcripts between the base gencode and de novo transcriptomes

225 (Supplemental Figure 3A), the per-sample Salmon mapping rate increased on average by

$2262.041 \%$ indicating that the vast majority of gene expression data is retained within our

227 transcriptome (Supplemental Figure 3B). 


\section{Novel Isoforms are identified in ocular tissues}

A

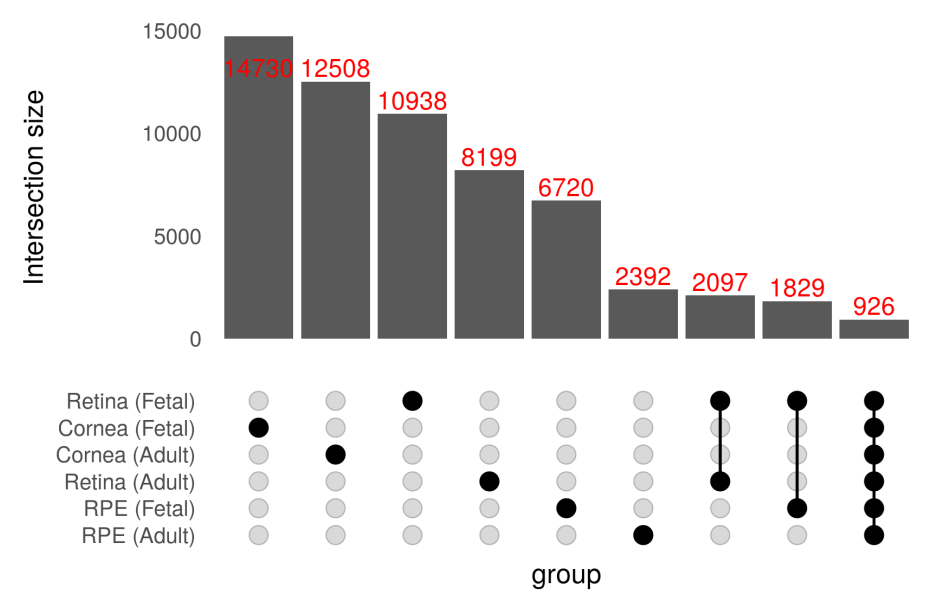

B

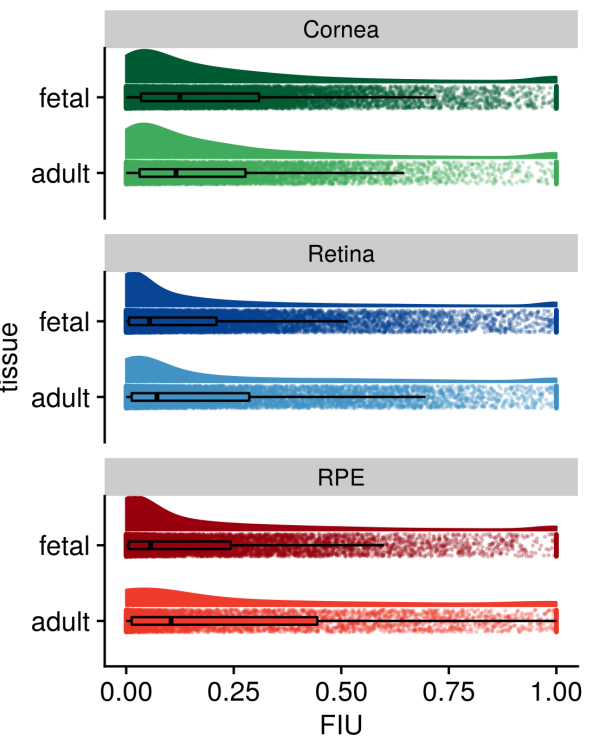

Figure 4. Overview of novel gene isoforms in the eye. A) Set intersection of novel isoforms in ocular transcriptomes. B) Boxplots of fraction isoform usage (FIU) overlaid over FIU data points with estimated distribution of data set above each boxplot.

Using the pan-eye transcriptome, we compared the overlap in constructed novel

isoforms across ocular subtissues and found that $77.968 \%$ of novel isoforms are specific to a singular ocular subtissue (Fig 4A). Additionally, fetal-like tissues had more novel isoforms that their adult counterpart. For each novel isoform we then calculated fraction isoform usage (FIU), or the fraction of total gene expression a transcript contributes to its parent gene. We found that, on average, novel isoforms contributed to $20.584 \%$ of their parent gene's expression but in each subtissue we found multiple novel isoforms that contribute to the majority of their parent genes expression (Fig 4B)

\section{Differential usage of gene isoforms occurs during retinal development}

Multiple studies have shown that gene isoforms play a significant role in eye

244 development (Bharti et al., 2008), (Mellough et al., 2019). We hypothesized that the DNTX

245 annotation provides additional insight into alternative isoform usage and identifies novel

246 gene isoforms potentially involved in eye development. We used RNA-seq data of the

247 developing retina from Mellough et al, an independent data set that we did not include for 
bioRxiv preprint doi: $\mathrm{https}$ //doi.org/10.1101/2020 $0821261644^{\cdot}$ this version posted September 22,2020 . The copyright holder for this preprint

(which was not certified by peer review) is the author/funder. This article is a US Government work. It is not subject to copyright under 17 USC 105 and is also made available for use under a CCO license.

248 transcriptome construction, and used a subset of the DNTX annotation corresponding to

249 fetal retina to quantify transcript expression and identify transcripts with significant

250 changes in expression across retinal development. Transcripts that are differentially

251 expressed (qvalue <.01) and have a mean FIU difference of .25 in at least one comparison of

252 time points are indicative of differential transcript usage (DTU). 
bioRxiv preprint doi: https://doi.org/10.1101/2020.08.21.261644; this version posted September 22, 2020. The copyright holder for this preprint (which was not certified by peer review) is the author/funder. This article is a US Government work. It is not subject to copyright under 17 USC 105 and is also made available for use under a CCO license.

A

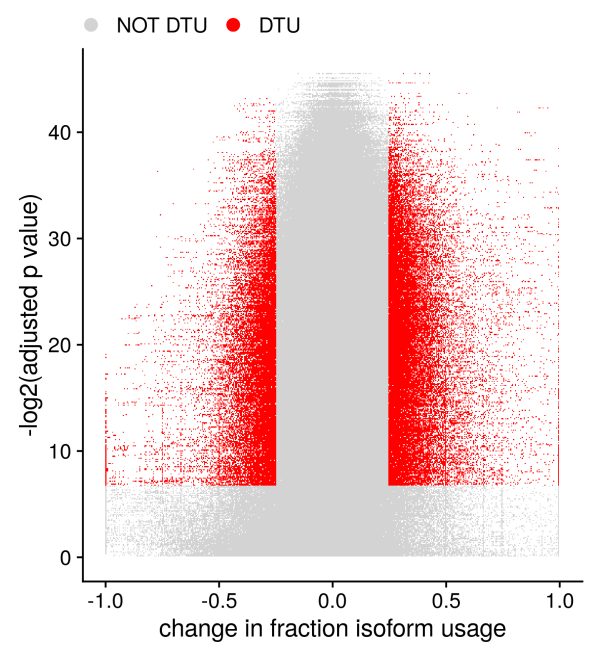

C

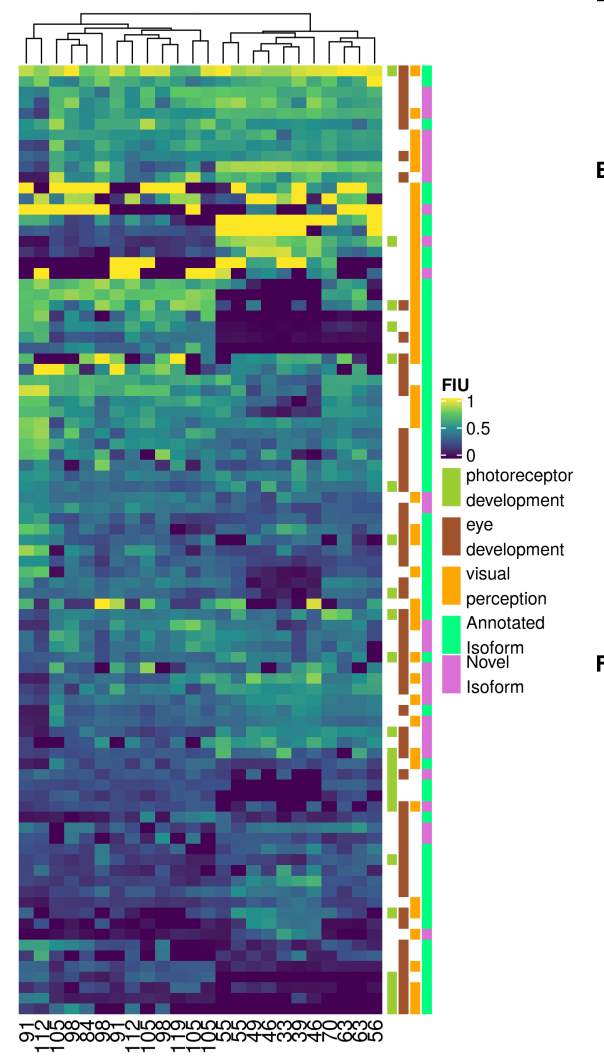

B development

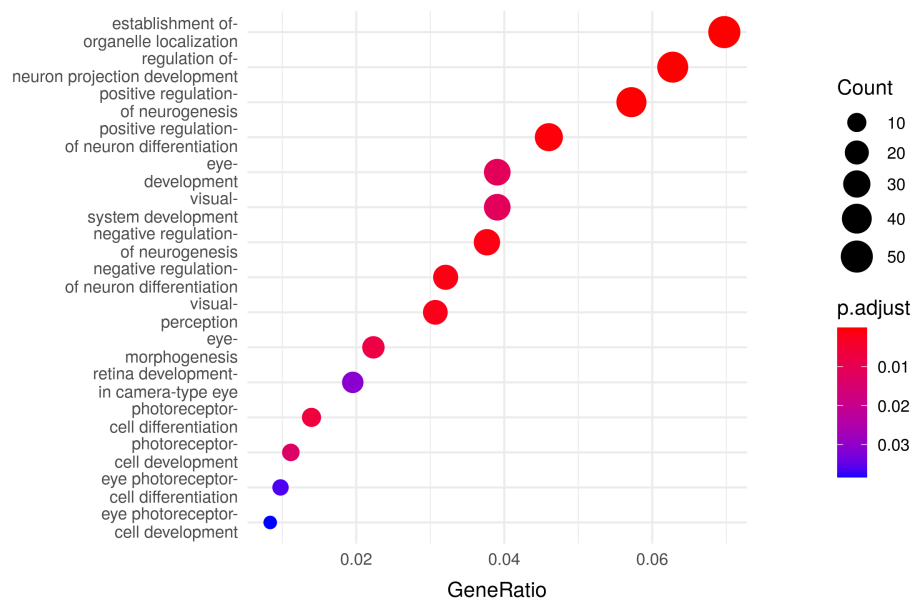

D

E
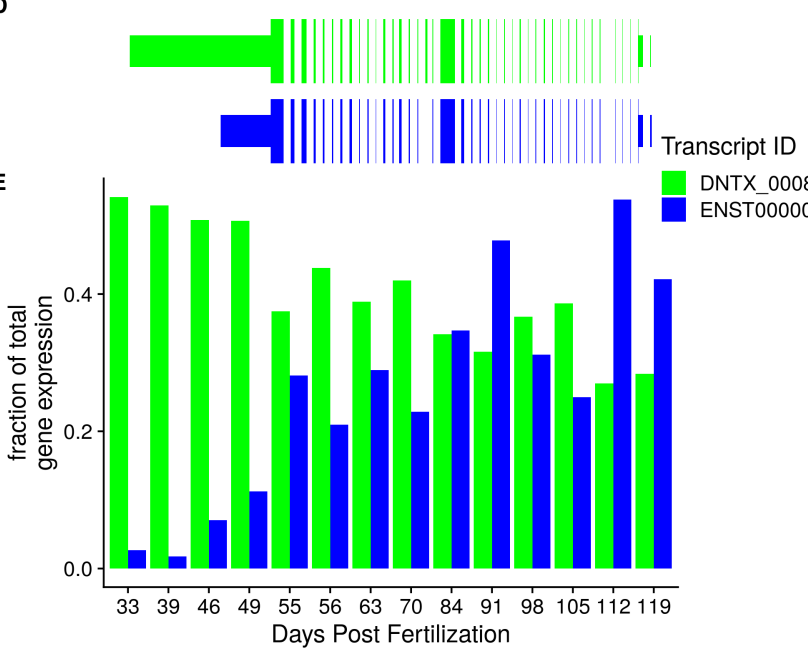

DNTX_00080076

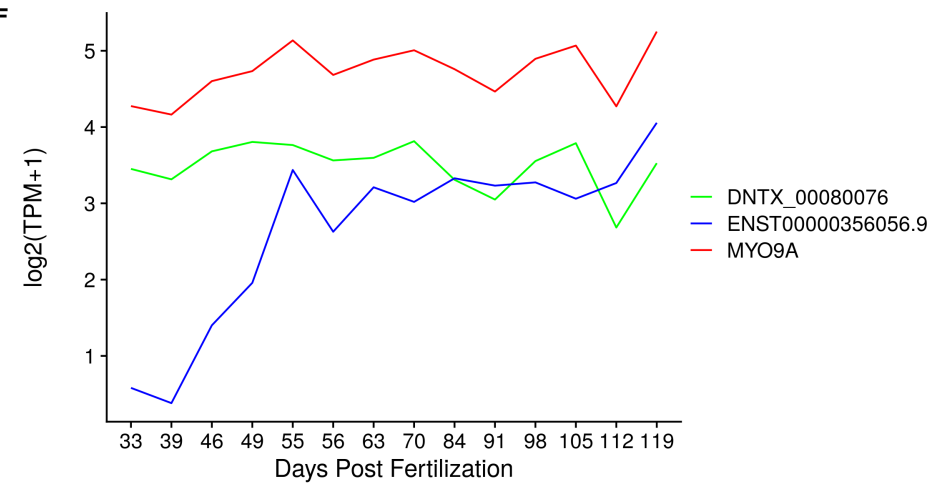

Figure 5 Differential Transcript usage during retinal development. A) Volcano plot of tested transcripts B) Dot plot for gene set enrichment analysis C) Heatmap of hiearchical clustering of transcripts with DTU associated with eye development D) Transcript models for MYO9A, a gene undergoing DTU E) FIU change in MYO9A FIU across development F) average log-transformed TPM expression of MYO9A across retinal 
We analyzed 24 samples across 14 developmental days post fertilization and found 1717 transcripts across 812 genes displaying DTU (Fig 5A). We found that genes involved in DTU are enriched(qvalue <.05) for genes related to eye and neurological development (Fig 5B), and that hierarchical clustering of DTU transcripts generates an early stage and late stage cluster (Fig 5C). One of these genes, MYO9A, is a classical example of DTU. MYO9A is associated with the visual perception GO term, plays a role in ocular development, and has been associated with ocular disease (Gorman et al., 1999). While expression of MYO9A remains relatively unchanged across development, expression of two of its associated isoforms in fetal retina (Fig 5D) changes dramatically during development: a novel isoform is highly expressed early during development, but switched to the canonical isoform later in development (Fig 5E,F). This novel isoform contains a novel exon within the protein coding region of the isoform as well as novel last exon extending the 3' UTR (Fig 5d). A full

273 list of genes and transcripts displaying DTU is available in Supplemental Data

274 (Supplemental Data 4).

\section{De novo transcriptomes allow for a more precise variant prioritization.}

The identification of a disease-causing variant through genome sequencing is a common step in diagnosing genetic disease, when disease causing variants cannot be determined from exonic sequencing. Prediction of a variant's biological impact and subsequent variant prioritization is a fundamental step in this process. Many methods for predicting variant effects on protein function or gene expression are based on location within the body of a transcript; for example variants that disrupt splice sites and start/stop codons are considered to be the most damaging, while variants within intronic and intergenic regions have unknown impact or are not classified, and, thus, are not included for further consideration. However, multiple studies have identified pathogenic deep intronic variants for retinal dystrophies (Braun et al., 2013), (Bauwens et al., 2019), (Zernant et al., 2014), (Sangermano et al., 2019), (Jamshidi et al., 2019), (Mayer et al., 2016), (Geoffroy et al., 2018). Pathogenic intronic variants are thought to function by introducing a novel splice site, disrupting regulatory motifs, or altering a tissue-specific

289 transcript. To explore this third possibility, we mapped known pathogenic intronic variants onto novel isoforms within the de novo transcriptomes. 


\begin{tabular}{|c|c|c|c|c|c|c|}
\hline $\begin{array}{r}\text { Gene } \\
\text { Name }\end{array}$ & $\begin{array}{r}\text { Associated } \\
\text { Disease }\end{array}$ & $\begin{array}{r}\text { Location } \\
\text { (hg19) }\end{array}$ & $\begin{array}{r}\text { Canonical } \\
\text { Variant } \\
\text { HGVS }\end{array}$ & $\begin{array}{r}\text { Gencode } \\
\text { Predicted } \\
\text { Consequence }\end{array}$ & $\begin{array}{r}\text { DNTX Predicted } \\
\text { Consequence }\end{array}$ & $\begin{array}{r}\text { Published } \\
\text { Study }\end{array}$ \\
\hline \multirow{7}{*}{$\mathrm{ABCA} 4$} & \multirow{2}{*}{$\begin{array}{r}\text { ABCA4- } \\
\text { associated } \\
\text { maculopathy }\end{array}$} & $\begin{array}{r}\text { Chr1:94481967 } \\
\text { C>T }\end{array}$ & $\begin{array}{r}\text { c.5197-557G>T, } \\
\text { NM_000350.2 }\end{array}$ & $\begin{array}{r}\text { intron variant, } \\
\text { downstream gene variant }\end{array}$ & 5 prime UTR variant & \multirow{2}{*}{ Bauwens et al } \\
\hline & & $\begin{array}{r}\text { Chr1:94546814 } \\
\text { G>C }\end{array}$ & $\begin{array}{r}\text { c.859-540C>G, } \\
\text { NM_000350.2 }\end{array}$ & intron variant & $\begin{array}{r}\text { non coding transcript } \\
\text { exon variant }\end{array}$ & \\
\hline & \multirow{5}{*}{$\begin{array}{r}\text { Stargardt } \\
\text { disease }\end{array}$} & $\begin{array}{r}\text { Chr1:94484001 } \\
\text { C>T }\end{array}$ & $\begin{array}{r}\text { C. } 5196+1137 G> \\
\text { A, NM_000350.2 }\end{array}$ & $\begin{array}{r}\text { intron variant, } \\
\text { downstream gene variant }\end{array}$ & 5 prime UTR variant & \multirow{2}{*}{$\begin{array}{l}\text { Braun et al } \\
\text { Zernant et al }\end{array}$} \\
\hline & & $\begin{array}{r}\text { Chr1:94484082 } \\
T>G\end{array}$ & $\begin{array}{r}\text { c.5196+1056A> } \\
\text { G, NM_000350.2 }\end{array}$ & $\begin{array}{r}\text { intron variant, } \\
\text { downstream gene variant }\end{array}$ & 5 prime UTR variant & \\
\hline & & $\begin{array}{r}\text { Chr1:94526934 } \\
T>G\end{array}$ & $\begin{array}{r}\text { c. } 1938-619 A>G, \\
\text { NM_000350.2 }\end{array}$ & $\begin{array}{r}\text { intron variant, } \\
\text { splice region variant, } \\
\text { non coding transcript } \\
\text { variant }\end{array}$ & $\begin{array}{r}\text { non coding transcript } \\
\text { exon variant }\end{array}$ & Zernant et al. \\
\hline & & $\begin{array}{r}\text { Chr1:94527698 } \\
\text { G>C }\end{array}$ & $\begin{array}{r}\text { c. } 1937+435 C>G, \\
\text { NM_000350.2 }\end{array}$ & $\begin{array}{r}\text { intron variant, } \\
\text { upstream gene variant }\end{array}$ & $\begin{array}{r}\text { non coding transcript } \\
\text { exon variant }\end{array}$ & \multirow{2}{*}{ Sangermano et al. } \\
\hline & & $\begin{array}{r}\text { Chr1:94546780 } \\
\text { C>G } \\
\end{array}$ & $\begin{array}{r}\text { c.859-506G }>C, \\
\text { NM_000350.2 }\end{array}$ & intron variant & $\begin{array}{r}\text { non coding transcript } \\
\text { exon variant }\end{array}$ & \\
\hline IFT140 & Ciliopathy & $\begin{array}{r}\text { Chr16:1576595 } \\
\text { C }>A\end{array}$ & $\begin{array}{r}\text { c. } 2577+25 G>A, \\
\text { NM_014714.3 }\end{array}$ & $\begin{array}{r}\text { upstream gene variant, } \\
\text { intron variant, } \\
\text { NMD transcript variant, } \\
\text { non coding transcript } \\
\text { exon variant, } \\
\text { non coding transcript } \\
\text { variant }\end{array}$ & missense variant & Geoffroy et al. \\
\hline PROM1 & $\begin{array}{l}\text { Cone-rod } \\
\text { dystrophy }\end{array}$ & $\begin{array}{r}\text { Chr4:15989860 } \\
T>G\end{array}$ & $\begin{array}{r}\text { c.2077-521A>G, } \\
\text { NM_006017.2 }\end{array}$ & $\begin{array}{r}\text { intron variant, } \\
\text { upstream gene variant }\end{array}$ & 5 prime UTR variant & Mayer et al. \\
\hline RPGRIP1 & $\begin{array}{r}\text { RPGRIP1- } \\
\text { mediated } \\
\text { inherited retinal } \\
\text { degeneration }\end{array}$ & $\begin{array}{r}\text { Chr14:21789588 } \\
\text { G>A }\end{array}$ & $\begin{array}{r}\text { c. } 1611+27 G>A \\
\text { NM_020366.3 }\end{array}$ & $\begin{array}{r}\text { intron variant, } \\
\text { non coding transcript } \\
\text { variant, } \\
\text { upstream gene variant, } \\
\text { synonymous variant, } \\
\text { NMD transcript variant, } \\
\text { downstream gene variant }\end{array}$ & 5 prime UTR variant & Jamshidi et al. \\
\hline
\end{tabular}

Table 2. Pathogenic variants previously considered intronic that are on expressed transcripts in the retina de novo transcriptome. Canonical human genome variation society (HGVS) annotation is based on transcripts from the RefSeq annnotation. Predicted consequences were generaed with the Variant Effect Predictor(VEP)

296 We used a list of 129 intronic and noncoding variants previously identified as

297 pathogenic for a retinal dystrophy and predicted the effect of these variants with Ensembl's

298 Variant Effect Predictor using a subset of the DNTX annotation corresponding to fetal and

299 adult retina as the input transcript annotation. We identified ten variants whose predicted

300 effect increased in severity due the presence of a novel gene isoform in a previously

301 intronic region (Table 2). Seven of these variants were in deep intronic hotpsots known for

302 pathogenic variation within the gene ABCA4. 


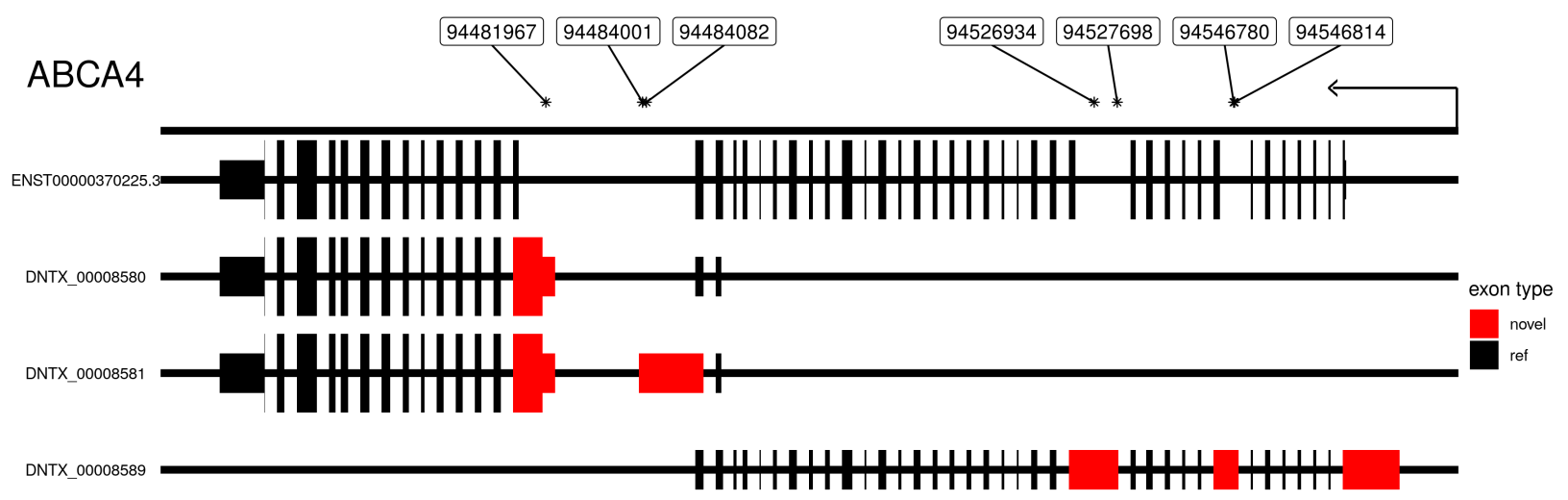

Figure 6. Transcript models for selected Isoforms of $A B C A 4$ along with location of pathogenic intronic variants. Location is on the hg19 human genome build. Thick lines indicate protein coding regions. Arrow indicates direction of transcription. Introns not drawn to scale

These variants were spanned by three distinct novel isoforms with two containing open reading frames (ORFs) encoding only the carboxy-terminus of the canonical protein

310 isoform, and one noncoding spanning the proximal half of the canonical isoform (Fig 6).

311 ABCA4 expression and function has also been observed in RPE (Lenis et al., 2018).

312 However, we did not observe these transcripts in RPE, suggesting that these pathogenic

313 variants are primarily affecting retinal-specific $A B C A 4$ transcripts. We note that these

314 transcripts have not been experimentally validated.

To further highlight the potential importance of de novo transcriptomes for future

316 genetic tests we determined how many genes associated with retinal disease from RetNet

317 have novel isoforms (sph.uth.edu/retnet/). We found that within the set of genes with

318 novel isoforms, there is significant enrichment of retinal disease genes (hypergeometric

319 pvalue $=3.4 \mathrm{e}-04)$, with 220 out of 379 RetNet genes having a novel isoform. A full list of

320 these genes is available in the Supplementary data(supplemental data 5). 


\section{A companion visualization tool enables easy use of de novo transcriptomes}

A

B

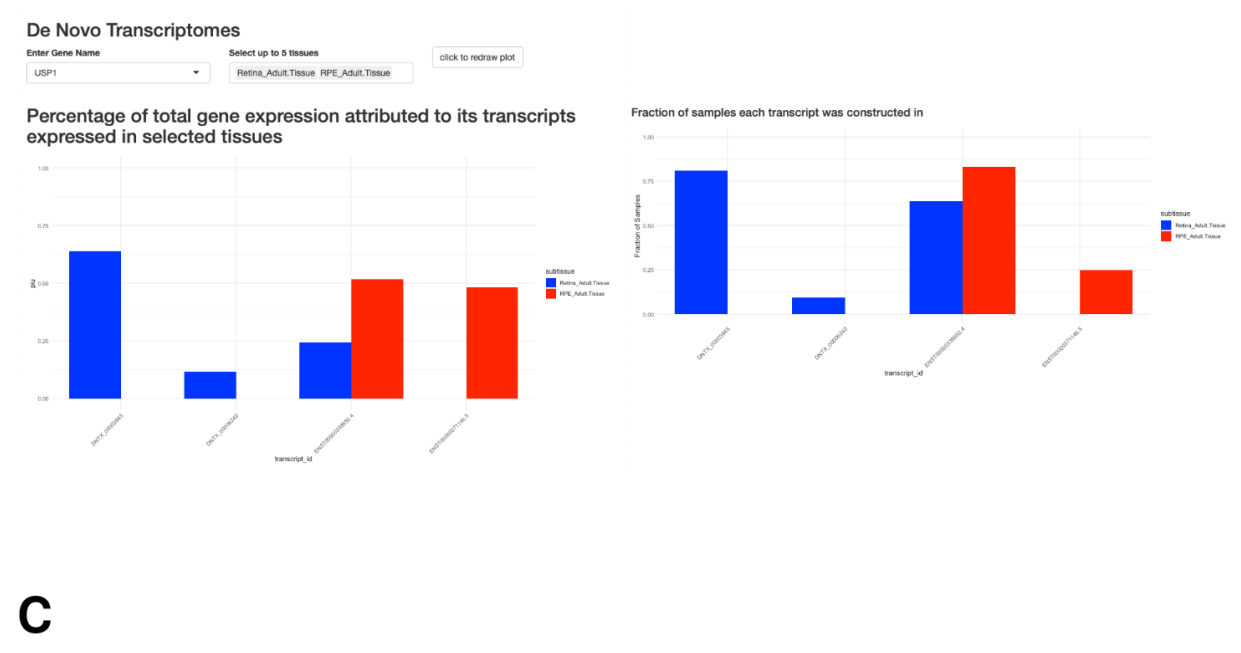

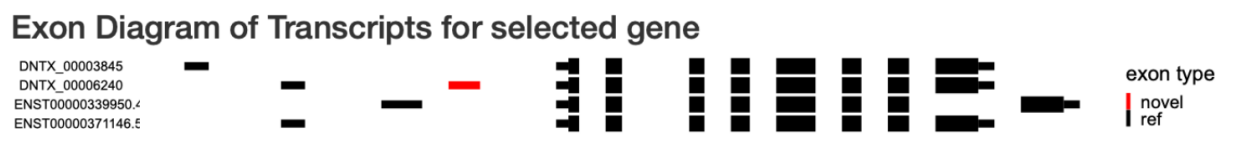

Figure 7. Screenshots from dynamic de novo transcriptome visualization tool. A). FIU bar plot for selected gene and subtissue. B). Exon level diagram of transcript body Thicklines represent coding region of transcript. novel exons colored in red. Tooltip contains genomic location and phylop score C) Bargraph of fraction of samples within dataset each transcript was consructed in by tissue. accessing our de novo transcriptomes. For each subtissue we show the FIU for each 
333

phylogenetic conservation score (Fig 7B). We additionally show a barplot of the fraction of samples each transcript was constructed in (Fig 7C). Users can also download the de novo transcriptomes for selected subtissues in GTF and fasta format. Instructions to download and run the app are available at https://github.com/vinayswamy/ocular_transcriptomes_shiny. While visualization of direct transcript expresion is not a part of this app, it can be viewed in the eyeIntegration app (Swamy and McGaughey, 2019) by selected 'DNTX' as the transcript annotation. Finally, we provide all code as a Snakemake workflow and provide a Docker container with all software required for the pipeline available at https://github.com/vinay-swamy/ocular_transcriptomes_pipeline

\section{Discussion}

Motivated by the lack of a comprehensive transcriptome for the eye, we constructed transcriptomes for adult and fetal retina, RPE and cornea. By using long-read RNA-seq data to calibrate our short-read construction pipeline, we were able to identify biologically relevant transcriptomes. We found that concordance between long and short-read-based transcriptomes is directly related to transcript length and transcript expression. We saw a clear inability within the PacBio data set to accurately detect transcripts shorter than $2000 \mathrm{bp}$ for both previously annotated and novel transcripts. As many of the transcripts constructed using short-reads are below this threshold, long-read sequencing data enriched for smaller transcript sizes would provide greater insight in future studies.

We used a large dataset compiled from published RNA-seq data to build the pan-eye transcriptomes, an approach that has several key advantages. First, the large sample size overcomes the noisy nature of RNA-seq data. Second, as the cohort is constructed from many independent studies, we are more confident that the transcriptomes accurately reflect the biology of their originating subtissue and are not a technical artifact due to preparation of the samples. As another line of evidence, the de novo transcriptomes match existing large scale data sets and are more conserved than existing annotations (Supplemental Figure 2). 
In each ocular subtissue we examined, we found hundreds of novel gene isoforms,

many of which were novel due to novel exons. Within ocular subtissues, these novel

isoforms are most commonly specific to single subtissue. This makes sense as a majority of the exons in our de novo transcriptomes are first and last exons, which have been

364 previously shown to significantly contribute to the tissue specificity of gene isoforms

365 (Reyes and Huber, 2018). We also found that on average novel isoforms represent about $20.584 \%$ of their parent gene's expression. Future studies are needed to identify the

367 function of these isoforms. One possibility is that some of these isoforms are only expressed in rare cell types, as transcript annotation was previously shown to be incomplete in rare cell types (Zhang et al., 2020). This especially makes sense in the retina which contain over a dozen distinct cell types, several of which contribute to $5 \%$ or less of the total cell population (Yan et al., 2020). As we imposed a strict expression filter as part of our transcriptome pipeline, we may have removed transcripts specific to rare cell types.

In conclusion, we created the first pan-eye transcriptome annotation and showed

that it is useful in understanding the role of gene isoforms in ocular biology and improving the ability to diagnose inherited eye diseases. We hope this work is useful as a starting point for other researchers; [delete] to make the transcriptomes easily accessible to other researchers we designed a webapp both for visualization and to quickly access tissuespecific annotation files. We believe this project will enable other researchers to explore new research directions and answer long pending questions.

\section{Methods}

381 Generation of PacBio long-read RNA sequencing data and Illumina short-read 382 RNA sequencing data

Human iPSCs were differentiated into RPE using previously described protocols in (Bryan et al., 2018) and (May-Simera et al., 2018). iPSC-derived RPE (iPSC-RPE) cells at 42 days post differentiation were lysed with TRIzol reagent (Thermo Fisher Scientific; cat \# 15596026) and total RNA was isolated using the Direct-zol RNA MiniPrep Kit (Zymo Research, Irvine, CA). 5-6 $\mu$ g total RNA that passed quality control metric (RIN >.9) were used for PacBio library preparation. For PacBio HiFi circular consensus sequencing(CCS), 
libraries were prepared following the "Procedure-Checklist-Iso-Seq-Express-Template-

Preparation-for-Sequel-and-Sequel-II-Systems" protocol. Two libraries were generated: one to capture transcripts 2 kilobases $(\mathrm{kb})$ or smaller, and one to capture transcripts

392 between 2-5kb. Sequencing was done on the PacBio Sequel II system for a movie time of 24 393 hours.

For Illumina sequencing, Poly-A selected stranded mRNA libraries were constructed from 0.5-1 $\mu$ g total RNA using the Illumina TruSeq Stranded mRNA Sample Prep Kits

396 according to manufacturer's instructions. Amplification was performed using 10-12 cycles

397 to minimize the risk of over-amplification. Unique dual-indexed barcode adapters were

398 applied to each library. Libraries were pooled in equimolar ratio and sequenced together

399 on a HiSeq 4000. At least 57 million 75-base read pairs were generated for each individual

400 library. Data was processed using illumina Real Time Analysis (RTA) version 2.7.7. All

401 library preparation and sequencing was performed at the National Institutes of Health

402 Intramural Sequencing Center (NISC).

403 Code availability and software versions.

404 To improve reproducibility, all code used for both the analyzing the data and 405 generating the figures for this paper was written as multiple Snakemake pipelines. Each

406 Snakefile contains the exact parameters for all tools and scripts used in each analysis.

407 (Köster and Rahmann, 2012) All code (and versions) used for this project is publicly

408 available in the following github repositories: https://github.com/vinay-

409 swamy/ocular_transcriptomes_pipeline (main pipeline), https://github.com/vinay-

410 swamy/ocular_transcriptomes_longread_analysis (long-read analysis pipeline),

411 https://github.com/vinay-swamy/ocular_transcriptomes_paper (figures and tables for this

412 paper), https://github.com/vinay-swamy/ocular_transcriptomes_shiny (webapp).

413 Additionally, all Snakefiles are included as supplementary data.(supplementary data files 1414 3)

\section{Analysis of long-read data}

416 PacBio sequencing movies were processed into full length, non-chimeric (FLNC)

417 reads using the IsoSeq3 3.1.2 pipeline in the PacBio SMRT link v7.0 software. The existing 
ENCODE long-read RNA-seq pipeline (https://github.com/ENCODE-DCC/long-read-rna-

419 pipeline) was rewritten as a Snakemake workflow as follows. Transcripts were aligned to

420 the human genome using minimap2(18), using an alignment index built on the gencode

421 v28 primary human genome. Sequencing errors in aligned long-reads were corrected using

422 TranscriptClean (19) with default parameters. Splice junctions for TranscriptClean were

423 obtained using the TranscriptClean accessory script "get_SJs_from_gtf.py" using the

424 gencode v28 comprehensive transcript annotation as the input. A list of common variants

425 to avoid correcting were obtained from the ENCODE portal

426 (https://www.encodeproject.org/files/ENCFF911UGW/). The long-read transcriptome

427 annotation was generated with TALON (20). A TALON database was generated using the

428 talon_initialize_database command, with all default parameters, except for the “-5P” and “-

4293 " parameters. These parameters represent the maximum distance between close 5' start

430 and 3' ends of similar transcript to merge and were both set to 100 to match parameters

431 used in later tools. Annotation in GTF format was generated using the talon_create_GTF

432 command, and transcript abundance values were generated using the talon_abundance

433 command.

\section{Analysis of short-read RPE data}

435 Each sample was aligned to the Gencode release 28 hg38 human genome assembly

436 using the genomic aligner STAR and the resulting BAM files were sorted using samtools

437 sort (Frankish et al., 2019),(Dobin et al., 2013),(Li et al., 2009). For each sorted BAM file, a

438 per-sample base transcriptome was constructed using StringTie with the Gencode v28

439 comprehensive annotation as a guiding annotation (Frankish et al., 2019),(Pertea et al.,

440 2015). All sample transcriptomes were merged with the long-read transcriptome using

441 gffcompare(Pertea and Pertea, 2020) with default parameters. We note that the default

442 values for the distance to merge similar 5' starts and 3 ends of transcripts in gffcompare is

443 the same to what we chose for TALON. We defined the metric construction accuracy, used

444 to evaluate short-read transcriptome construction as the following:

445 Construction Accuracy $=\frac{\text { short read transcriptome } \cap \text { long read transcriptome }}{\text { short read transcriptome }}$ 


\section{Construction of subtissue-specific transcriptomes.}

We constructed transcriptomes for 1217 samples in the Eye in a Disk(EiaD), a dataset generated from aggregating publically available healthy, unperturbed RNA-seq samples from 50 distinct locations of the body across 29 different studies. Specific information on how this dataset was generated is detailed in the methods from our previous work (Swamy and McGaughey, 2019). We constructed a transcriptome for each

452 sample, and merged samples together to create 50 subtissue-specific transcriptomes. We 453 define subtissue as a unique body location and are either temporally different versions of 454 the same tissue(adult vs fetal tissue), or different regions of a larger tissue (cortex vs 455 cerebellum in brain). Tissue refers to complete whole tissue (retina, brain, liver). For each 456 subtissue-specific transcriptome, we removed transcripts that had an average expression

457 less than 1 Transcripts Per Million (TPM) across all samples of the same subtissue type. All subtissue-specific transcriptomes were merged to form a single unified annotation file in general transfer format(GTF) to ensure transcript identifiers were the same across

460 subtissues. We merged all ocular subtissue transcriptomes to generate a separate pan-eye 461 transcriptome.

\section{Subtissue specific transcriptome quantification}

For each resulting subtissue specific transcriptome, we extracted transcript sequences using the tool gffread and used these sequences to build a subtissue-specific quantification index using the index mode of the alignment-free quantification tool Salmon (Pertea and Pertea, 2020), (Patro et al., 2017). For each sample, we quantified transcript expression using the quant mode of Salmon, using a sample's respective subtissue specific quantification index. We similarly quantified all ocular samples using the pan-eye

469 transcriptome and the Gencode v28 reference transcriptome.

\section{Annotation of novel exons}

First, a comprehensive set of distinct, annotated exons was generated by merging

472 exon annotation from gencode, ensembl, UCSC, and refseq. We then defined a novel exon as 473 any exon within our transcriptomes that does not exactly match the chromosome, start, 
categories: first, last, and middle exons. We then extracted all annotated exon start and stop

sites from our set of previously annotated exons. Novel middle exons that have an annotated start but an unannotated end were categorized as a novel alternative 3' end exons and similarly novel middle exons with an unannotated start but annotated end were categorized as a novel alternative 5' start exons. Novel middle exons whose start and end match annotated exon start and ends were considered retained introns. Novel middle exons whose start and end do not match annotated starts and ends were considered fully novel exons. We then classified novel first and last exons. Novel first exons were first exons whose start is not in the set of annotated exon starts, and novel last exons were terminal exons whose end is not in the set of annotated exon ends. This analysis of novel transcripts is implemented in our Rscript “annotate_and_make_tissue_gtfs.R”.

\section{Validation of DNTX with phylop, CAGE data, and polyA signals}

PhyloP scores for the phylop 20-way multi species alignment were downloaded from UCSC's FTP server on October 16th, 2019 and converted from bigWig format to bed format using the wig2bed tool in BEDOPs (Pollard et al., 2010), (Neph et al., 2012). The average score per exon in both the gencode and DNTX annotation was calculated by

491 intersecting exon locations with phylop scores and then averaging the per base score for

492 each exon, using the intersect and groupby tools from the bedtools suite, respectively.

493 Significant difference in mean phylop score was tested with a Mann Whitney U test.

CAGE peaks were download from the FANTOM FTP server

(https://fantom.gsc.riken.jp/5/datafiles/reprocessed/hg38_latest/extra/CAGE_peaks/hg3

496 8_fair+new_CAGE_peaks_phase1and2.bed.gz) on June 15th 2020 (Noguchi et al., 2017).

497 Transcriptional start sites (TSS) were extracted from gencode and DNTX annotations; TSS

498 is defined as the start of the first exon of a transcript. Distance to CAGE peaks was

499 calculated using the closest tool in the bedtools suite. Significant difference in mean

500 distance to CAGE peak between DNTX and gencode annotation was tested with a Mann

501 Whitney U test. 
504 .bed.gz) on June 15th 2020 (Herrmann et al., 2020). Transcriptional end sites(TES) were 505 extracted from gencode and DNTX annotations; TES is defined as the end of the terminal 506 exon of a transcript. Distance to polyA signal was calculated using the closest tool in the 507 bedtools suite (Quinlan and Hall, 2010). Significant difference in mean distance to polyA 508 signal was tested with a Mann Whitney U test.

\section{Identification of novel protein coding transcripts}

$510 \quad$ Protein-coding transcripts in the unified transcriptome were identified using the

511 TransDecoder suite (Haas et al., 2013). Transcript sequences in fasta format were extracted

512 from the final pan-body transcriptome using the TransDecoder util script

513 “gtf_genome_to_cdna_fasta.pl”. Potential open reading frames(ORFs) were generated from

514 transcript sequences using the LongestORF module within TransDecoder, and the single

515 best ORF for each transcript was extracted with the Predict module within Transdecoder.

516 The resulting ORFs were mapped to genomic locations with the TransDecoder util script

517 "gtf_to_alignment_gff3.pl". For each ORF start and stop codons were extracted with the

518 script "agat_sp_add_start_stop.pl" scripts from the AGAT toolkit

519 (https://github.com/NBISweden/AGAT/). Transcripts with no detectable ORF or missing a 520 start or stop codon were labelled as noncoding.

\section{Analysis of novel isoforms in eye tissues}

$522 \quad$ An Upset plot was generated using the ComplexUpset package

523 (https://github.com/krassowski/complex-upset) (Lex et al., 2014). Fraction Isoform Usage

524 (FIU) was calculated for each transcript $t$ associated with a parent gene $g$ using the

525 following formula: $F I U_{t}=\frac{T P M_{t}}{T P M_{g}}$. Raincloud plots of FIU were generated using the

526 “R_Rainclouds" R package (Allen et al., 2019).

\section{Analysis of fetal retina RNA-seq data.}

528 RNA-seq samples from Mellough et al. were obtained from EiaD, and were not

529 included in the main dataset used for building transcriptomes. Outliers within the dataset

530 were identified by first performing principal component analysis of transcript level

531 expression data, calculating the center of all data using the first two principal components, 
532 and subsequently removing five samples furthest away from the center of all data. The

533 remaining samples were normalized using calcNormFactors from the R package edgeR and

534 converted to weights using the voom function from the R package limma (Robinson et al.,

535 2010), (Ritchie et al., 2015). Differential expression was modeled using the lmFit function

536 using developmental time point as the model design and tested for significant change in

537 expression using the Ebayes function from limma. Gene Set enrichment was tested using

538 the R package clusterprofileR (Yu et al., 2012). Heatmaps were generated using the

539 ComplexHeatmap package (Gu et al., 2016).

540 Prediction of variant impact using de novo transcriptomes.

$541 \quad$ Noncoding variants previously associated with retinal disease from the Blueprint

542 Genetics Retinal dystrophy panel were obtained from the Blueprint Genetics website

543 (https://blueprintgenetics.com/tests/panels/ophthalmology/retinal-dystrophy-panel/).

544 The variants were converted from HGVS to VCF format using a custom python script

545 "HGVS_to_VCF.py". This VCF was then remapped to the hg38 human genome build using

546 the tool crossmap (Zhao et al., 2014). The VCF of variants was used as the input variants for

547 the Variant Effect Predictor(VEP) tool from Ensembl, with each subtissue specific

548 transcriptome as the input annotation (McLaren et al., 2016). VEP was additionally run

549 using the gencode v28 comprehensive annotation as the input annotation to identify

550 variants whose predicted impact increased in severity.

551 Figures, Tables, and Computing Resources

$552 \quad$ All statistical analyses, figures and tables in this paper were generated using the $\mathrm{R}$ 553 programming language. (R Core Team, 2019) A full list of packages and versions can be

554 found in the supplementary file session_info.txt. All computation was performed on the 555 National Institutes of Health high performance computer system Biowulf (hpc.nih.gov).

\section{Competing Interests}

$557 \quad$ All authors declare no Competing interests. 
bioRxiv preprint doi: https://doi.org/10.1101/2020.08.21.261644; this version posted September 22, 2020. The copyright holder for this preprint (which was not certified by peer review) is the author/funder. This article is a US Government work. It is not subject to copyright under 17 USC 105 and is also made available for use under a CCO license.

\section{Supplemental Figures}

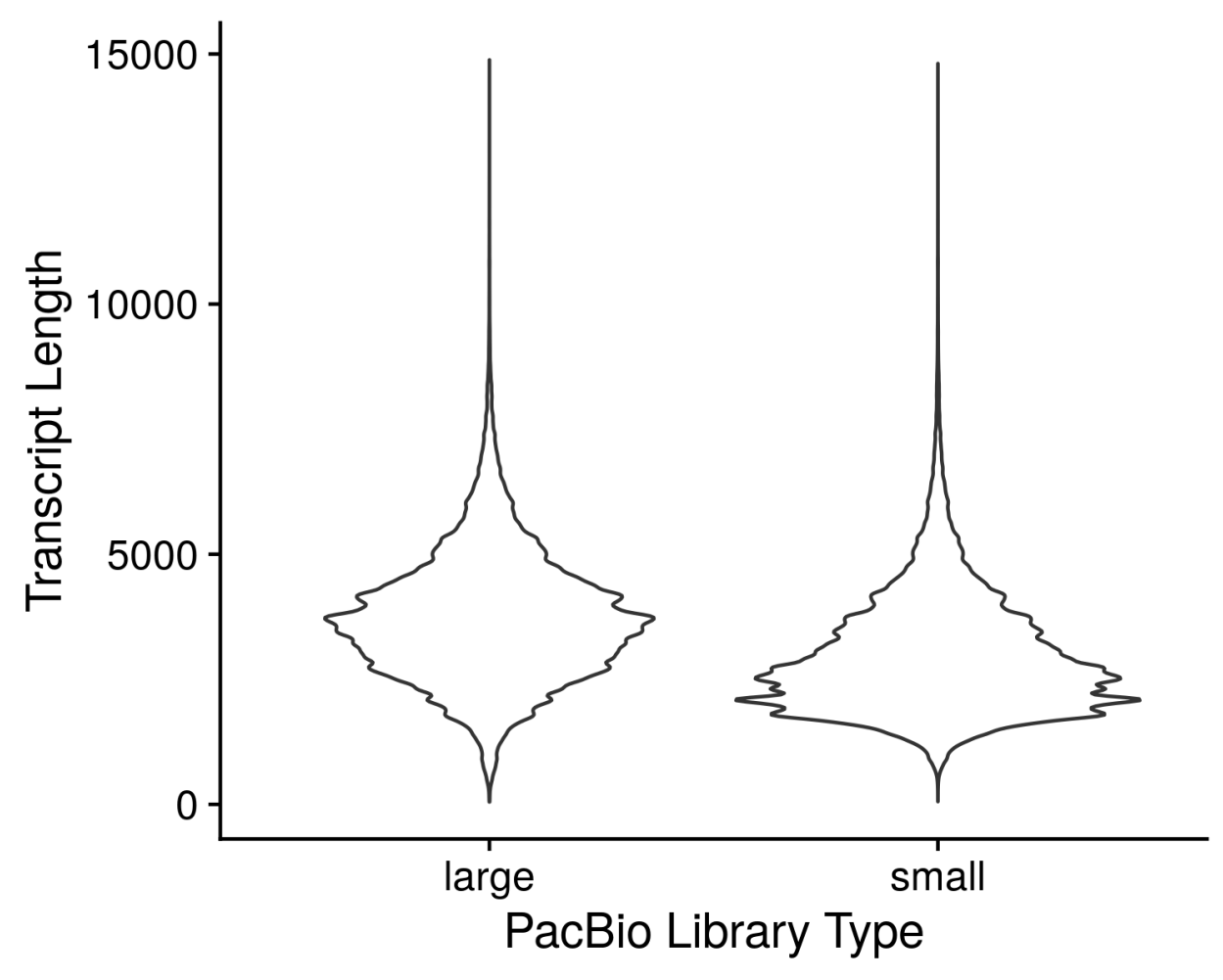
library sizes. 
bioRxiv preprint doi: https://doi.org/10.1101/2020.08.21.261644; this version posted September 22, 2020. The copyright holder for this preprint (which was not certified by peer review) is the author/funder. This article is a US Government work. It is not subject to copyright under 17 USC 105 and is also made available for use under a CCO license.
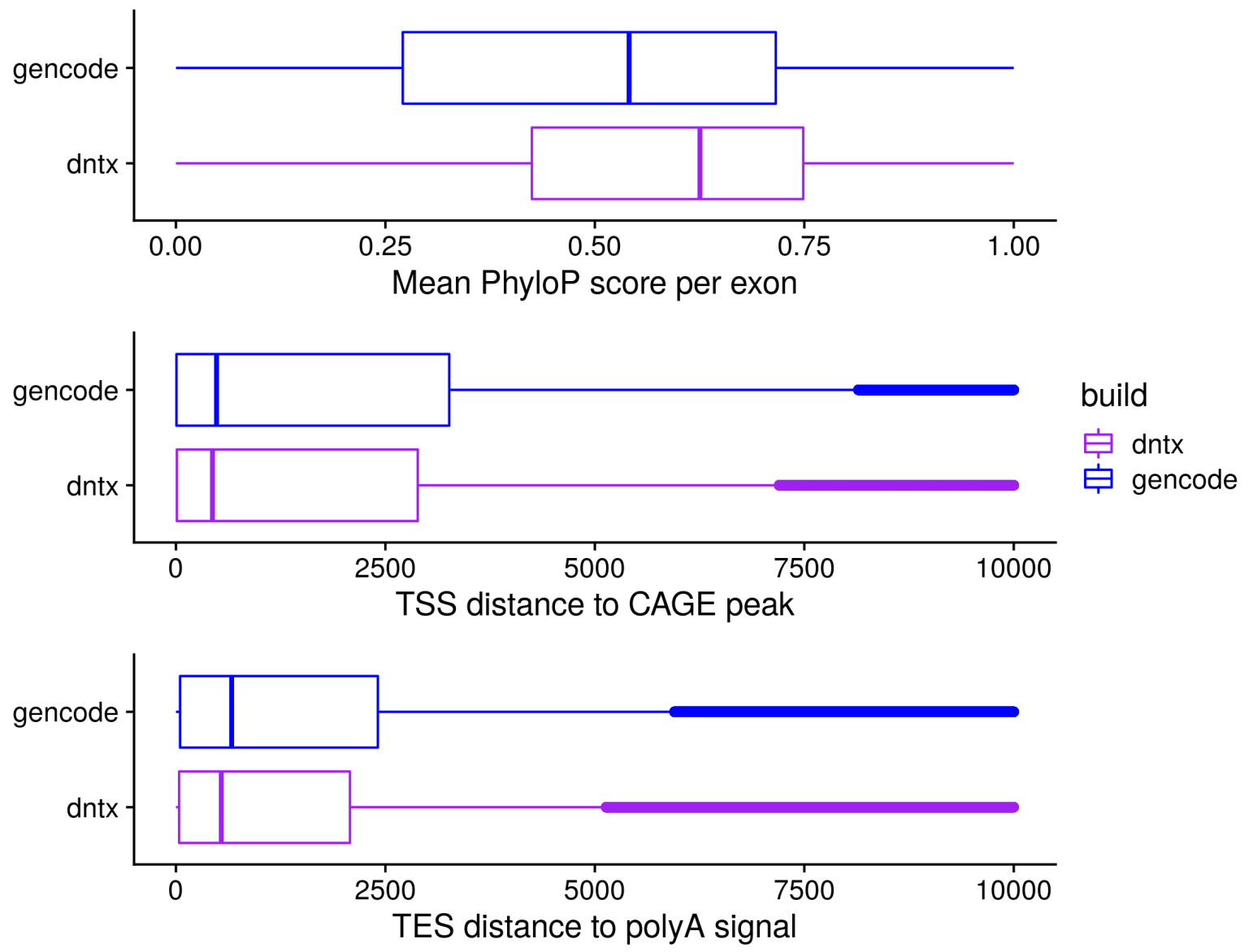

Supplemental Figure 2. Comparison of DNTX annotation to Gencode annotation. A) Average per exon Phylop score for Gencode and DNTX transcripts. B) Average distance of DNTX transcriptional start sites (TSS) and Gencode TSS to CAGE-seq peaks from the FANTOM consortium. C) Average distance of DNTX transcriptional end sites (TES) and Gencode TES to polyadenylation signals in the PolyA site atlas. 

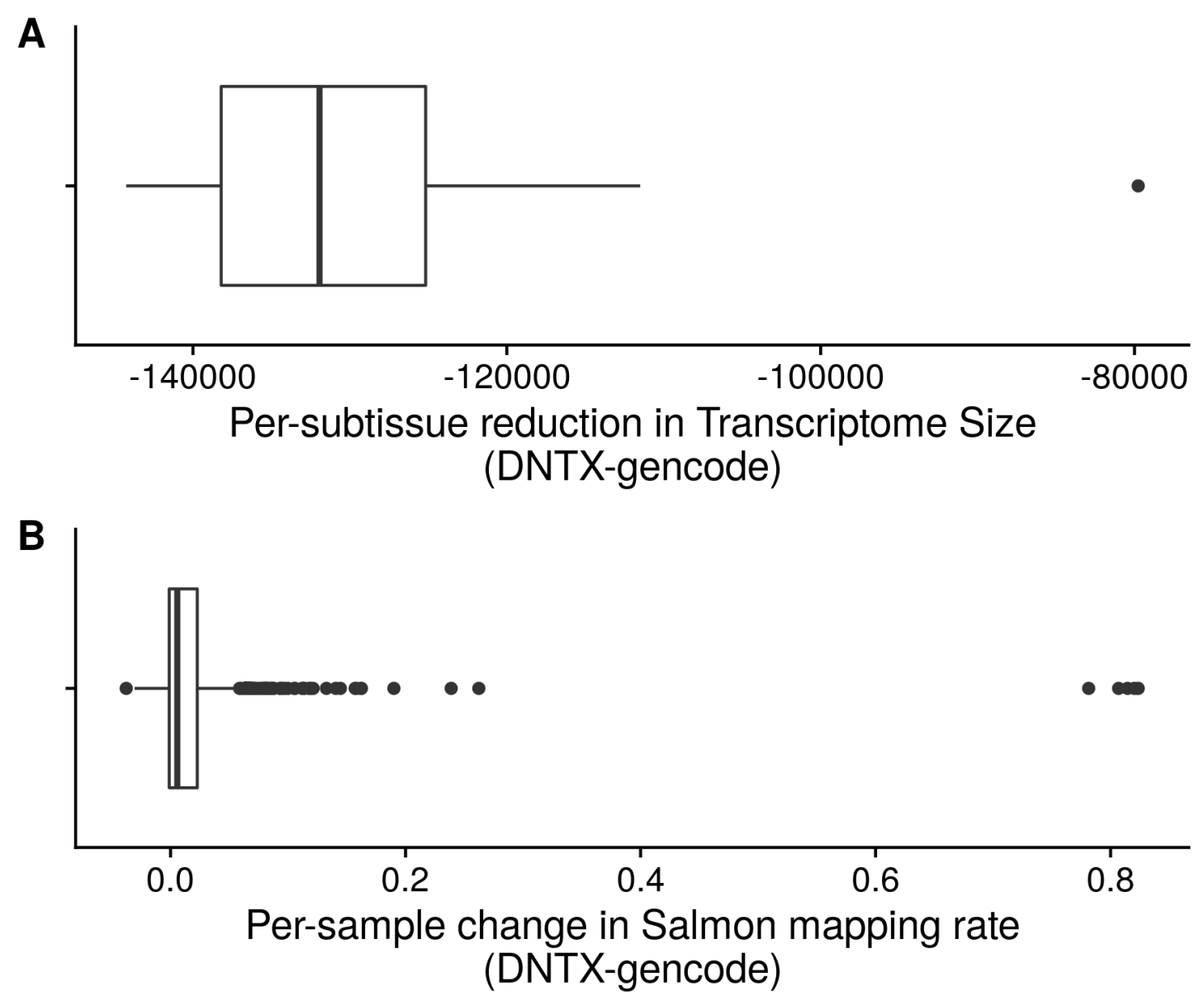

\section{References}

573 Allen M, Poggiali D, Whitaker K, Marshall TR, Kievit RA. 2019. Raincloud plots: A multi-

574 platform tool for robust data visualization. Wellcome Open Research 4:63.

575 doi:10.12688/wellcomeopenres.15191.1

576 Bauwens M, Garanto A, Sangermano R, Naessens S, Weisschuh N, De Zaeytijd J, Khan M,

577 Sadler F, Balikova I, Van Cauwenbergh C, Rosseel T, Bauwens J, De Leeneer K, De Jaegere S,

578 Van Laethem T, De Vries M, Carss K, Arno G, Fakin A, Webster AR, Ravel de l'Argentière TJL

579 de, Sznajer Y, Vuylsteke M, Kohl S, Wissinger B, Cherry T, Collin RWJ, Cremers FPM, Leroy

580 BP, De Baere E. 2019. ABCA4-associated disease as a model for missing heritability in

581 autosomal recessive disorders: Novel noncoding splice, cis-regulatory, structural, and

582 recurrent hypomorphic variants. Genetics in Medicine 21:1761-1771. doi:10.1038/s41436-

583 018-0420-y 
584

585

586

587

588

589

590

591

592

593

594

595

596

597

598

599

600

601

602

603

604

605

606

607

608

609

610

611

612

613

614

615

616

617

618

619

620

621

622

Beck AH, Weng Z, Witten DM, Zhu S, Foley JW, Lacroute P, Smith CL, Tibshirani R, Rijn M van de, Sidow A, West RB. 2010. 3'-End Sequencing for Expression Quantification (3SEQ) from Archival Tumor Samples. PLOS ONE 5:e8768. doi:10.1371/journal.pone.0008768

Bharti K, Liu W, Csermely T, Bertuzzi S, Arnheiter H. 2008. Alternative promoter use in eye development: Complex role and regulation of the transcription factor MITF. Development (Cambridge, England) 135:1169-1178. doi:10.1242/dev.014142

Blenkinsop TA, Saini JS, Maminishkis A, Bharti K, Wan Q, Banzon T, Lotfi M, Davis J, Singh D, Rizzolo LJ, Miller S, Temple S, Stern JH. 2015. Human Adult Retinal Pigment Epithelial Stem Cell-Derived RPE Monolayers Exhibit Key Physiological Characteristics of Native Tissue. Investigative Ophthalmology \& Visual Science 56:7085-7099. doi:10.1167/iovs.14-16246

Braun TA, Mullins RF, Wagner AH, Andorf JL, Johnston RM, Bakall BB, Deluca AP, Fishman GA, Lam BL, Weleber RG, Cideciyan AV, Jacobson SG, Sheffield VC, Tucker BA, Stone EM. 2013. Non-exomic and synonymous variants in ABCA4 are an important cause of Stargardt disease. Human Molecular Genetics 22:5136-5145. doi:10.1093/hmg/ddt367

Bryan JM, Fufa TD, Bharti K, Brooks BP, Hufnagel RB, McGaughey DM. 2018. Identifying core biological processes distinguishing human eye tissues with precise systems-level gene expression analyses and weighted correlation networks. Human Molecular Genetics 27:3325-3339. doi:10.1093/hmg/ddy239

Dobin A, Davis CA, Schlesinger F, Drenkow J, Zaleski C, Jha S, Batut P, Chaisson M, Gingeras TR. 2013. STAR: Ultrafast universal RNA-seq aligner. Bioinformatics (Oxford, England) 29:15-21. doi:10.1093/bioinformatics/bts635

Dykes IM, Bueren KL van, Scambler PJ. 2018. HIC2 regulates isoform switching during maturation of the cardiovascular system. Journal of Molecular and Cellular Cardiology 114:29-37. doi:10.1016/j.yjmcc.2017.10.007

Frankish A, Diekhans M, Ferreira A-M, Johnson R, Jungreis I, Loveland J, Mudge JM, Sisu C, Wright J, Armstrong J, Barnes I, Berry A, Bignell A, Carbonell Sala S, Chrast J, Cunningham F, Di Domenico T, Donaldson S, Fiddes IT, Garcia Giron C, Gonzalez JM, Grego T, Hardy M, Hourlier T, Hunt T, Izuogu OG, Lagarde J, Martin FJ, Martinez L, Mohanan S, Muir P, Navarro FCP, Parker A, Pei B, Pozo F, Ruffier M, Schmitt BM, Stapleton E, Suner M-M, Sycheva I, Uszczynska-Ratajczak B, Xu J, Yates A, Zerbino D, Zhang Y, Aken B, Choudhary JS, Gerstein M, Guigo R, Hubbard TJP, Kellis M, Paten B, Reymond A, Tress ML, Flicek P. 2019. GENCODE reference annotation for the human and mouse genomes. Nucleic acids research 47:D766D773. doi:10.1093/nar/gky955

Geoffroy V, Stoetzel C, Scheidecker S, Schaefer E, Perrault I, Bär S, Kröll A, Delbarre M, Antin M, Leuvrey A-S, Henry C, Blanché H, Decker E, Kloth K, Klaus G, Mache C, Martin-Coignard D, McGinn S, Boland A, Deleuze J-F, Friant S, Saunier S, Rozet J-M, Bergmann C, Dollfus H, Muller J. 2018. Whole-genome sequencing in patients with ciliopathies uncovers a novel recurrent tandem duplication in IFT140. Human Mutation 39:983-992.

doi:10.1002/humu.23539 
623 Gorman SW, Haider NB, Grieshammer U, Swiderski RE, Kim E, Welch JW, Searby C, Leng S,

624 Carmi R, Sheffield VC, Duhl DM. 1999. The Cloning and Developmental Expression of Unconventional Myosin IXA (MY09A) a Gene in the Bardet-Biedl Syndrome (BBS4) Region at Chromosome 15q22-q23. Genomics 59:150-160. doi:10.1006/geno.1999.5867

627 GTEx Consortium, Laboratory, Data Analysis \&Coordinating Center (LDACC)—Analysis Working Group, Statistical Methods groups-Analysis Working Group, Enhancing GTEx (eGTEx) groups, NIH Common Fund, NIH/NCI, NIH/NHGRI, NIH/NIMH, NIH/NIDA, Biospecimen Collection Source Site-NDRI, Biospecimen Collection Source Site-RPCI, Biospecimen Core Resource-VARI, Brain Bank Repository-University of Miami Brain Endowment Bank, Leidos Biomedical-Project Management, ELSI Study, Genome Browser Data Integration \&Visualization-EBI, Genome Browser Data Integration \&VisualizationUCSC Genomics Institute, University of California Santa Cruz, Lead analysts: Laboratory, Data Analysis \&Coordinating Center (LDACC): NIH program management: Biospecimen collection: Pathology: eQTL manuscript working group: Battle A, Brown CD, Engelhardt BE, Montgomery SB. 2017. Genetic effects on gene expression across human tissues. Nature 550:204-213. doi:10.1038/nature 24277

Gu Z, Eils R, Schlesner M. 2016. Complex heatmaps reveal patterns and correlations in multidimensional genomic data. Bioinformatics 32:2847-2849. doi:10.1093/bioinformatics/btw313

Haas BJ, Papanicolaou A, Yassour M, Grabherr M, Blood PD, Bowden J, Couger MB, Eccles D, Li B, Lieber M, MacManes MD, Ott M, Orvis J, Pochet N, Strozzi F, Weeks N, Westerman R, William T, Dewey CN, Henschel R, LeDuc RD, Friedman N, Regev A. 2013. De novo transcript sequence reconstruction from RNA-Seq: Reference generation and analysis with Trinity. Nature protocols 8. doi:10.1038/nprot.2013.084

647 Herrmann CJ, Schmidt R, Kanitz A, Artimo P, Gruber AJ, Zavolan M. 2020. PolyASite 2.0: A 648 consolidated atlas of polyadenylation sites from $3^{\prime}$ end sequencing. Nucleic Acids Research 649 48:D174-D179. doi:10.1093/nar/gkz918

650 Jamshidi F, Place EM, Mehrotra S, Navarro-Gomez D, Maher M, Branham KE, Valkanas E, 651 Cherry TJ, Lek M, MacArthur D, Pierce EA, Bujakowska KM. 2019. Contribution of non652 coding mutations to RPGRIP1-mediated inherited retinal degeneration. Genetics in medicine : official journal of the American College of Medical Genetics 21:694-704.

654 doi:10.1038/s41436-018-0104-7

655 Klimanskaya I, Hipp J, Rezai KA, West M, Atala A, Lanza R. 2004. Derivation and comparative assessment of retinal pigment epithelium from human embryonic stem cells using transcriptomics. Cloning and Stem Cells 6:217-245. doi:10.1089/clo.2004.6.217

658 Köster J, Rahmann S. 2012. Snakemake-a scalable bioinformatics workflow engine. 659 Bioinformatics 28:2520-2522. doi:10.1093/bioinformatics/bts480

660 Landry J-R, Mager DL, Wilhelm BT. 2003. Complex controls: The role of alternative 661 promoters in mammalian genomes. Trends in Genetics 19:640-648. 
663 Lenis TL, Hu J, Ng SY, Jiang Z, Sarfare S, Lloyd MB, Esposito NJ, Samuel W, Jaworski C, Bok D, 664 Finnemann SC, Radeke MJ, Redmond TM, Travis GH, Radu RA. 2018. Expression of ABCA4 665 in the retinal pigment epithelium and its implications for Stargardt macular degeneration. 666 Proceedings of the National Academy of Sciences 115:E11120-E11127.

667 doi:10.1073/pnas.1802519115

668 Lex A, Gehlenborg N, Strobelt H, Vuillemot R, Pfister H. 2014. UpSet: Visualization of 669 Intersecting Sets. IEEE Transactions on Visualization and Computer Graphics 20:1983-1992. 670 doi:10.1109/TVCG.2014.2346248

671 Li H, Handsaker B, Wysoker A, Fennell T, Ruan J, Homer N, Marth G, Abecasis G, Durbin R, 6721000 Genome Project Data Processing Subgroup. 2009. The Sequence Alignment/Map 673 format and SAMtools. Bioinformatics (Oxford, England) 25:2078-2079.

674 doi:10.1093/bioinformatics/btp352

675 Maruotti J, Sripathi SR, Bharti K, Fuller J, Wahlin KJ, Ranganathan V, Sluch VM, Berlinicke CA, Davis J, Kim C, Zhao L, Wan J, Qian J, Corneo B, Temple S, Dubey R, Olenyuk BZ, Bhutto I, Lutty GA, Zack DJ. 2015. Small-molecule-directed, efficient generation of retinal pigment epithelium from human pluripotent stem cells. Proceedings of the National Academy of Sciences 112:10950-10955. doi:10.1073/pnas.1422818112

680 Mayer AK, Rohrschneider K, Strom TM, Glöckle N, Kohl S, Wissinger B, Weisschuh N. 2016. Homozygosity mapping and whole-genome sequencing reveals a deep intronic PROM1 mutation causing cone-rod dystrophy by pseudoexon activation. European Journal of

683 Human Genetics 24:459-462. doi:10.1038/ejhg.2015.144 May-Simera HL, Wan Q, Jha BS, Hartford J, Khristov V, Dejene R, Chang J, Patnaik S, Lu Q, Banerjee P, Silver J, Insinna-Kettenhofen C, Patel D, Lotfi M, Malicdan M, Hotaling N, Maminishkis A, Sridharan R, Brooks B, Miyagishima K, Gunay-Aygun M, Pal R, Westlake C, Miller S, Sharma R, Bharti K. 2018. Primary Cilium-Mediated Retinal Pigment Epithelium Maturation Is Disrupted in Ciliopathy Patient Cells. Cell reports 22:189-205.

McLaren W, Gil L, Hunt SE, Riat HS, Ritchie GRS, Thormann A, Flicek P, Cunningham F.

691 2016. The Ensembl Variant Effect Predictor. Genome Biology 17:122. doi:10.1186/s13059-

692 016-0974-4

693 Mellough CB, Bauer R, Collin J, Dorgau B, Zerti D, Dolan DWP, Jones CM, Izuogu OG, Yu M, 694 Hallam D, Steyn JS, White K, Steel DH, Santibanez-Koref M, Elliott DJ, Jackson MS, Lindsay S, Grellscheid S, Lako M. 2019. An integrated transcriptional analysis of the developing 696 human retina. Development (Cambridge, England) 146. doi:10.1242/dev.169474

697 Mills JD, Nalpathamkalam T, Jacobs HIL, Janitz C, Merico D, Hu P, Janitz M. 2013. RNA-Seq 698 analysis of the parietal cortex in Alzheimer's disease reveals alternatively spliced isoforms 699 related to lipid metabolism. Neuroscience Letters 536:90-95.

700 doi:10.1016/j.neulet.2012.12.042 
Mitra M, Lee HN, Coller HA. 2020. Splicing Busts a Move: Isoform Switching Regulates Migration. Trends in Cell Biology 30:74-85. doi:10.1016/j.tcb.2019.10.007

703

704

705

706

707

708

709

710

711

712

713

714

715

716

717

718

719

720

721

722

723

724

725

726

727

728

729

730

731

732

733

734

735

736

737

738

739

740

741

742
Nagalakshmi U, Wang Z, Waern K, Shou C, Raha D, Gerstein M, Snyder M. 2008. The Transcriptional Landscape of the Yeast Genome Defined by RNA Sequencing. Science 320:1344-1349. doi:10.1126/science.1158441

Neagoe Ciprian, Kulke Michael, del Monte Federica, Gwathmey Judith K., de Tombe Pieter P., Hajjar Roger J., Linke Wolfgang A. 2002. Titin Isoform Switch in Ischemic Human Heart Disease. Circulation 106:1333-1341. doi:10.1161/01.CIR.0000029803.93022.93

Neph S, Kuehn MS, Reynolds AP, Haugen E, Thurman RE, Johnson AK, Rynes E, Maurano MT, Vierstra J, Thomas S, Sandstrom R, Humbert R, Stamatoyannopoulos JA. 2012. BEDOPS: High-performance genomic feature operations. Bioinformatics 28:1919-1920. doi:10.1093/bioinformatics/bts277

Noguchi S, Arakawa T, Fukuda S, Furuno M, Hasegawa A, Hori F, Ishikawa-Kato S, Kaida K, Kaiho A, Kanamori-Katayama M, Kawashima T, Kojima M, Kubosaki A, Manabe R-i, Murata M, Nagao-Sato S, Nakazato K, Ninomiya N, Nishiyori-Sueki H, Noma S, Saijyo E, Saka A, Sakai M, Simon C, Suzuki N, Tagami M, Watanabe S, Yoshida S, Arner P, Axton RA, Babina M, Baillie JK, Barnett TC, Beckhouse AG, Blumenthal A, Bodega B, Bonetti A, Briggs J, Brombacher F, Carlisle AJ, Clevers HC, Davis CA, Detmar M, Dohi T, Edge ASB, Edinger M, Ehrlund A, Ekwall K, Endoh M, Enomoto H, Eslami A, Fagiolini M, Fairbairn L, FarachCarson MC, Faulkner GJ, Ferrai C, Fisher ME, Forrester LM, Fujita R, Furusawa J-i, Geijtenbeek TB, Gingeras T, Goldowitz D, Guhl S, Guler R, Gustincich S, Ha TJ, Hamaguchi M, Hara M, Hasegawa Y, Herlyn M, Heutink P, Hitchens KJ, Hume DA, Ikawa T, Ishizu Y, Kai C, Kawamoto H, Kawamura YI, Kempfle JS, Kenna TJ, Kere J, Khachigian LM, Kitamura T, Klein S, Klinken SP, Knox AJ, Kojima S, Koseki H, Koyasu S, Lee W, Lennartsson A, Mackay-sim A, Mejhert N, Mizuno Y, Morikawa H, Morimoto M, Moro K, Morris KJ, Motohashi H, Mummery CL, Nakachi Y, Nakahara F, Nakamura T, Nakamura Y, Nozaki T, Ogishima S, Ohkura N, Ohno H, Ohshima M, Okada-Hatakeyama M, Okazaki Y, Orlando V, Ovchinnikov DA, Passier R, Patrikakis M, Pombo A, Pradhan-Bhatt S, Qin X-Y, Rehli M, Rizzu P, Roy S, Sajantila A, Sakaguchi S, Sato H, Satoh H, Savvi S, Saxena A, Schmidl C, Schneider C, Schulze-Tanzil GG, Schwegmann A, Sheng G, Shin JW, Sugiyama D, Sugiyama T, Summers KM, Takahashi N, Takai J, Tanaka H, Tatsukawa H, Tomoiu A, Toyoda H, Wetering M van de, Berg LM van den, Verardo R, Vijayan D, Wells CA, Winteringham LN, Wolvetang E, Yamaguchi Y, Yamamoto M, Yanagi-Mizuochi C, Yoneda M, Yonekura Y, Zhang PG, Zucchelli S, Abugessaisa I, Arner E, Harshbarger J, Kondo A, Lassmann T, Lizio M, Sahin S, Sengstag T, Severin J, Shimoji H, Suzuki M, Suzuki H, Kawai J, Kondo N, Itoh M, Daub CO, Kasukawa T, Kawaji H, Carninci P, Forrest ARR, Hayashizaki Y. 2017. FANTOM5 CAGE profiles of human and mouse samples. Scientific Data 4:170112. doi:10.1038/sdata.2017.112

O'Leary NA, Wright MW, Brister JR, Ciufo S, Haddad D, McVeigh R, Rajput B, Robbertse B, Smith-White B, Ako-Adjei D, Astashyn A, Badretdin A, Bao Y, Blinkova O, Brover V, Chetvernin V, Choi J, Cox E, Ermolaeva O, Farrell CM, Goldfarb T, Gupta T, Haft D, Hatcher E, Hlavina W, Joardar VS, Kodali VK, Li W, Maglott D, Masterson P, McGarvey KM, Murphy MR, O’Neill K, Pujar S, Rangwala SH, Rausch D, Riddick LD, Schoch C, Shkeda A, Storz SS, Sun H, 
743

744

745

746

747

748

749

750

751

752

753

754

755

756

757

758

759

760

761

762

763

764

765

766

767

768

769

770

771

772

773

774

775

776

777

778

779

Thibaud-Nissen F, Tolstoy I, Tully RE, Vatsan AR, Wallin C, Webb D, Wu W, Landrum MJ, Kimchi A, Tatusova T, DiCuccio M, Kitts P, Murphy TD, Pruitt KD. 2016. Reference sequence (RefSeq) database at NCBI: Current status, taxonomic expansion, and functional annotation. Nucleic Acids Research 44:D733-745. doi:10.1093/nar/gkv1189

Patro R, Duggal G, Love MI, Irizarry RA, Kingsford C. 2017. Salmon provides fast and biasaware quantification of transcript expression. Nature methods 14:417-419. doi:10.1038/nmeth.4197

Perrin RM, Konopatskaya O, Qiu Y, Harper S, Bates DO, Churchill AJ. 2005. Diabetic retinopathy is associated with a switch in splicing from anti- to pro-angiogenic isoforms of vascular endothelial growth factor. Diabetologia 48:2422-2427. doi:10.1007/s00125-0051951-8

Pertea G, Pertea M. 2020. GFF Utilities: GffRead and GffCompare. F1000Research 9:304. doi:10.12688/f1000research.23297.1

Pertea M, Kim D, Pertea GM, Leek JT, Salzberg SL. 2016. Transcript-level expression analysis of RNA-seq experiments with HISAT, StringTie and Ballgown. Nature Protocols 11:1650-1667. doi:10.1038/nprot.2016.095

Pertea M, Pertea GM, Antonescu CM, Chang T-C, Mendell JT, Salzberg SL. 2015. StringTie enables improved reconstruction of a transcriptome from RNA-seq reads. Nature Biotechnology 33:290-295. doi:10.1038/nbt.3122

Pertea M, Shumate A, Pertea G, Varabyou A, Breitwieser FP, Chang Y-C, Madugundu AK, Pandey A, Salzberg SL. 2018. CHESS: A new human gene catalog curated from thousands of large-scale RNA sequencing experiments reveals extensive transcriptional noise. Genome Biology 19:208. doi:10.1186/s13059-018-1590-2

Pollard KS, Hubisz MJ, Rosenbloom KR, Siepel A. 2010. Detection of nonneutral substitution rates on mammalian phylogenies. Genome Research 20:110-121.

doi:10.1101/gr.097857.109

Quinlan AR, Hall IM. 2010. BEDTools: A flexible suite of utilities for comparing genomic features. Bioinformatics (Oxford, England) 26:841-842.

doi:10.1093/bioinformatics/btq033

R Core Team. 2019. R: A Language and Environment for Statistical Computing. Vienna, Austria: R Foundation for Statistical Computing.

Reyes A, Huber W. 2018. Alternative start and termination sites of transcription drive most transcript isoform differences across human tissues. Nucleic Acids Research 46:582-592. doi:10.1093/nar/gkx1165

Ritchie ME, Phipson B, Wu D, Hu Y, Law CW, Shi W, Smyth GK. 2015. Limma powers differential expression analyses for RNA-sequencing and microarray studies. Nucleic Acids Research 43:e47-e47. doi:10.1093/nar/gkv007 
Robinson MD, McCarthy DJ, Smyth GK. 2010. edgeR: A Bioconductor package for differential expression analysis of digital gene expression data. Bioinformatics 26:139-140. doi:10.1093/bioinformatics/btp616

783

784

785

786

787

788

789

790

791

792

793

794

795

796

797

798

799

800

801

802

803

804

805

806

807

808

809

810

811

812

813

814

815

Sangermano R, Garanto A, Khan M, Runhart EH, Bauwens M, Bax NM, Born LI van den, Khan MI, Cornelis SS, Verheij JBGM, Pott J-WR, Thiadens AAHJ, Klaver CCW, Puech B, Meunier I, Naessens S, Arno G, Fakin A, Carss KJ, Raymond FL, Webster AR, Dhaenens C-M, Stöhr H, Grassmann F, Weber BHF, Hoyng CB, De Baere E, Albert S, Collin RWJ, Cremers FPM. 2019. Deep-intronic ABCA4 variants explain missing heritability in Stargardt disease and allow correction of splice defects by antisense oligonucleotides. Genetics in Medicine 21:17511760. doi:10.1038/s41436-018-0414-9

Swamy V, McGaughey D. 2019. Eye in a Disk: eyeIntegration Human Pan-Eye and Body Transcriptome Database Version 1.0. Investigative Ophthalmology \& Visual Science 60:3236-3246. doi:10.1167/iovs.19-27106

Takahashi H, Kato S, Murata M, Carninci P. 2012. CAGE- Cap Analysis Gene Expression: A protocol for the detection of promoter and transcriptional networks. Methods in molecular biology (Clifton, NJ) 786:181-200. doi:10.1007/978-1-61779-292-2_11

Tian B, Manley JL. 2017. Alternative polyadenylation of mRNA precursors. Nature Reviews Molecular Cell Biology 18:18-30. doi:10.1038/nrm.2016.116

Trapnell C, Williams BA, Pertea G, Mortazavi A, Kwan G, Baren MJ van, Salzberg SL, Wold BJ, Pachter L. 2010. Transcript assembly and quantification by RNA-Seq reveals unannotated transcripts and isoform switching during cell differentiation. Nature Biotechnology 28:511515. doi:10.1038/nbt.1621

Vitting-Seerup K, Sandelin A. 2017. The Landscape of Isoform Switches in Human Cancers. Molecular Cancer Research 15:1206-1220. doi:10.1158/1541-7786.MCR-16-0459

Wang Y, Liu J, HUANG B, XU Y-M, LI J, HUANG L-F, LIN J, ZHANG J, MIN Q-H, YANG W-M, WANG X-Z. 2015. Mechanism of alternative splicing and its regulation. Biomedical Reports 3:152-158. doi:10.3892/br.2014.407

Wenger AM, Peluso P, Rowell WJ, Chang P-C, Hall RJ, Concepcion GT, Ebler J, Fungtammasan A, Kolesnikov A, Olson ND, Töpfer A, Alonge M, Mahmoud M, Qian Y, Chin CS, Phillippy AM, Schatz MC, Myers G, DePristo MA, Ruan J, Marschall T, Sedlazeck FJ, Zook JM, Li H, Koren S, Carroll A, Rank DR, Hunkapiller MW. 2019. Accurate circular consensus long-read sequencing improves variant detection and assembly of a human genome. Nature Biotechnology 37:1155-1162. doi:10.1038/s41587-019-0217-9

Yan W, Peng Y-R, Zyl T van, Regev A, Shekhar K, Juric D, Sanes JR. 2020. Cell Atlas of The Human Fovea and Peripheral Retina. Scientific Reports 10:9802. doi:10.1038/s41598-02066092-9 
816 Yu G, Wang L-G, Han Y, He Q-Y. 2012. clusterProfiler: An R Package for Comparing

817 Biological Themes Among Gene Clusters. OMICS : a Journal of Integrative Biology 16:284-

818 287. doi:10.1089/omi.2011.0118

819 Zerbino DR, Achuthan P, Akanni W, Amode MR, Barrell D, Bhai J, Billis K, Cummins C, Gall A,

820 Girón CG, Gil L, Gordon L, Haggerty L, Haskell E, Hourlier T, Izuogu OG, Janacek SH,

821 Juettemann T, To JK, Laird MR, Lavidas I, Liu Z, Loveland JE, Maurel T, McLaren W, Moore B,

822 Mudge J, Murphy DN, Newman V, Nuhn M, Ogeh D, Ong CK, Parker A, Patricio M, Riat HS,

823 Schuilenburg H, Sheppard D, Sparrow H, Taylor K, Thormann A, Vullo A, Walts B, Zadissa A,

824 Frankish A, Hunt SE, Kostadima M, Langridge N, Martin FJ, Muffato M, Perry E, Ruffier M,

825 Staines DM, Trevanion SJ, Aken BL, Cunningham F, Yates A, Flicek P. 2018. Ensembl 2018.

826 Nucleic Acids Research 46:D754-D761. doi:10.1093/nar/gkx1098

827 Zernant J, Xie Y(, Ayuso C, Riveiro-Alvarez R, Lopez-Martinez M-A, Simonelli F, Testa F, 828 Gorin MB, Strom SP, Bertelsen M, Rosenberg T, Boone PM, Yuan B, Ayyagari R, Nagy PL, 829 Tsang SH, Gouras P, Collison FT, Lupski JR, Fishman GA, Allikmets R. 2014. Analysis of the 830 ABCA4 genomic locus in Stargardt disease. Human Molecular Genetics 23:6797-6806. 831 doi:10.1093/hmg/ddu396

832 Zhang D, Guelfi S, Garcia-Ruiz S, Costa B, Reynolds RH, D'Sa K, Liu W, Courtin T, Peterson A, 833 Jaffe AE, Hardy J, Botía JA, Collado-Torres L, Ryten M. 2020. Incomplete annotation has a 834 disproportionate impact on our understanding of Mendelian and complex neurogenetic 835 disorders. Science Advances 6:eaay8299. doi:10.1126/sciadv.aay8299

836 Zhao H, Sun Z, Wang J, Huang H, Kocher J-P, Wang L. 2014. CrossMap: A versatile tool for 837 coordinate conversion between genome assemblies. Bioinformatics (Oxford, England)

838 30:1006-1007. doi:10.1093/bioinformatics/btt730 